Mineral Resources Program

Systems-Deposits-Commodities-Critical Minerals Table for the Earth Mapping Resources Initiative

Open-File Report 2020-1042

Version 1.1, May 2021 



\section{Systems-Deposits-Commodities-Critical Minerals Table for the Earth Mapping Resources Initiative}

By Albert H. Hofstra and Douglas C. Kreiner

Mineral Resources Program

Open-File Report 2020-1042

Version 1.1, May 2021 


\section{U.S. Geological Survey, Reston, Virginia \\ First release: 2020 \\ Revised: May 2021 (ver 1.1)}

For more information on the USGS - the Federal source for science about the Earth, its natural and living resources, natural hazards, and the environment—visit https://www.usgs.gov or call 1-888-ASK-USGS.

For an overview of USGS information products, including maps, imagery, and publications, visit https://store.usgs.gov/.

Any use of trade, firm, or product names is for descriptive purposes only and does not imply endorsement by the U.S. Government.

Although this information product, for the most part, is in the public domain, it also may contain copyrighted materials as noted in the text. Permission to reproduce copyrighted items must be secured from the copyright owner.

Suggested citation:

Hofstra, A.H., and Kreiner, D.C., 2020, Systems-Deposits-Commodities-Critical Minerals Table for the Earth Mapping Resources Initiative (ver. 1.1, May 2021): U.S. Geological Survey Open-File Report 2020-1042, 26 p.,

https://doi.org/10.3133/ofr20201042.

ISSN 2331-1258 (online) 


\section{Acknowledgments}

This report benefited from discussions with numerous Mineral Resources Program personnel. We thank Nora Foley (U.S. Geological Survey [USGS]), William Lassetter (Virginia Geological Survey), and Lukas Zurcher (USGS) for early reviews of the Systems-Deposits-CommoditiesCritical Minerals Table, as well as Laurel Woodruff (USGS) and Jamey Jones (USGS) for their constructive reviews of this report. 



\section{Contents}

Acknowledgments ……...................................................................................................................

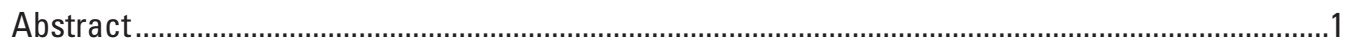

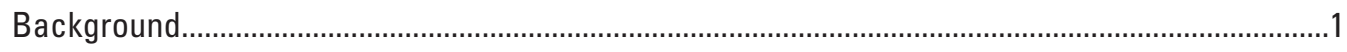

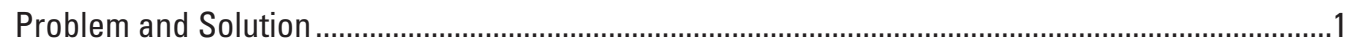

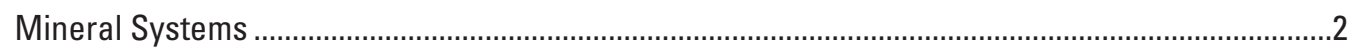

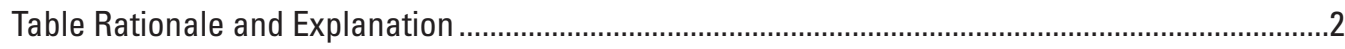

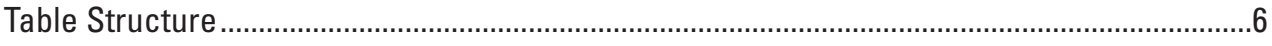

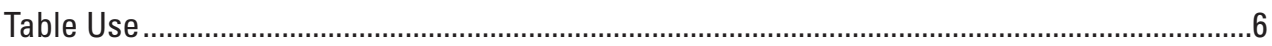

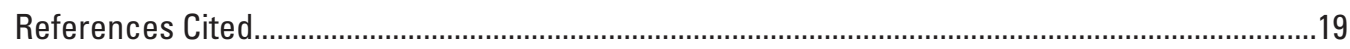

\section{Figures}

1. Diagrams showing mineral system concepts....................................................................

2. Schematic cross sections of a porphyry copper-molybdenum-gold system at

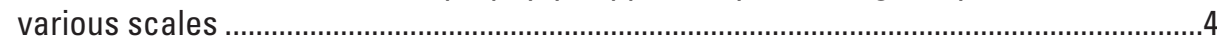

3. Schematic model of a basin brine path system.............................................................

\section{Table}

1. Systems-Deposits-Commodities-Critical Minerals Table for the Earth Mapping Resources Initiative.

\section{Conversion Factors}

International System of Units to U.S. customary units

\begin{tabular}{|c|c|c|}
\hline Multiply & By & To obtain \\
\hline \multicolumn{3}{|c|}{ Length } \\
\hline kilometer $(\mathrm{km})$ & 0.6214 & mile (mi) \\
\hline \multicolumn{3}{|c|}{ Area } \\
\hline square kilometer $\left(\mathrm{km}^{2}\right)$ & 0.3861 & square mile $\left(\mathrm{mi}^{2}\right)$ \\
\hline
\end{tabular}

\section{Abbreviations}

Earth MRI Earth Mapping Resources Initiative

PGE platinum group elements

REE rare earth elements

USGS U.S. Geological Survey 


\section{Chemical Symbols}

\begin{tabular}{|c|c|}
\hline $\mathrm{Ag}$ & silver \\
\hline $\mathrm{Al}$ & aluminum \\
\hline $\mathrm{Ag}$ & silver \\
\hline $\mathrm{Al}$ & aluminum \\
\hline As & arsenic \\
\hline $\mathrm{Au}$ & gold \\
\hline $\mathrm{BaSO}_{4}$ & barite \\
\hline $\mathrm{Be}$ & beryllium \\
\hline $\mathrm{Bi}$ & bismuth \\
\hline C & graphite \\
\hline $\mathrm{CaF}_{2}$ & fluorspar \\
\hline Co & cobalt \\
\hline $\mathrm{Cr}$ & chromium \\
\hline Cs & cesium \\
\hline $\mathrm{Cu}$ & copper \\
\hline $\mathrm{Fe}$ & iron \\
\hline $\mathrm{Ga}$ & gallium \\
\hline $\mathrm{Ge}$ & germanium \\
\hline $\mathrm{He}$ & helium \\
\hline $\mathrm{Hf}$ & hafnium \\
\hline In & indium \\
\hline $\mathrm{KCl}$ & potash \\
\hline $\mathrm{Li}$ & lithium \\
\hline $\mathrm{Mg}$ & magnesium \\
\hline $\mathrm{Mn}$ & manganese \\
\hline Mo & molybdenum \\
\hline $\mathrm{Nb}$ & niobium \\
\hline $\mathrm{Ni}$ & nickel \\
\hline$P$ & phosphorus \\
\hline $\mathrm{Pb}$ & lead \\
\hline $\mathrm{Rb}$ & rubidium \\
\hline $\operatorname{Re}$ & rhenium \\
\hline $\mathrm{Sb}$ & antimony \\
\hline $\mathrm{Sc}$ & scandium \\
\hline
\end{tabular}




$\begin{array}{ll}\mathrm{Sn} & \text { tin } \\ \mathrm{Sr} & \text { strontium } \\ \mathrm{Ta} & \text { tantalum } \\ \mathrm{Te} & \text { tellurium } \\ \mathrm{Ti} & \text { titanium } \\ \mathrm{U} & \text { uranium } \\ \mathrm{V} & \text { vanadium } \\ \mathrm{W} & \text { tungsten } \\ \mathrm{Zn} & \text { zinc } \\ \mathrm{Zr} & \text { zirconium }\end{array}$





\title{
Systems-Deposits-Commodities-Critical Minerals Table for the Earth Mapping Resources Initiative
}

\author{
By Albert H. Hofstra and Douglas C. Kreiner
}

\section{Abstract}

To define and prioritize focus areas across the United States with resource potential for 35 critical minerals in a few years' time, the U.S Geological Survey Earth Mapping Resources Initiative (Earth MRI) required an efficient approach to streamline workflow. A mineral systems approach based on current understanding of how ore deposits that contain critical minerals form and relate to broader geologic frameworks and the tectonic history of the Earth was used to satisfy this Earth MRI need. This report describes the rationale for, and structure of, a table developed for Earth MRI that relates critical minerals and principal commodities to the deposit types and mineral systems in which they are concentrated. The hierarchical relationship between systems, deposits, commodities, and critical minerals makes it possible to define and prioritize each system-based focus area once for all of the critical minerals that it may contain. This approach is advantageous because mineral systems are much larger than individual ore deposits and they generally have geologic features that can be "imaged" by the topographic, geologic, geochemical, and geophysical mapping techniques deployed by Earth MRI.

\section{Background}

The President and Secretary of the Interior issued orders (Executive Office of the President, 2017; U.S. Department of the Interior, 2017) that directed the U.S. Geological Survey (USGS) to develop a plan to improve the Nation's understanding of domestic critical mineral resources. In response, a list of 35 critical minerals with a high risk for supply disruption were identified by the National Minerals Information Center (Fortier and others, 2018). The 35 critical minerals that were identified are aluminum (Al), antimony ( $\mathrm{Sb}$ ), arsenic (As), barite $\left(\mathrm{BaSO}_{4}\right)$, beryllium $(\mathrm{Be})$, bismuth $(\mathrm{Bi})$, cesium $(\mathrm{Cs})$, chromium $(\mathrm{Cr})$, cobalt $(\mathrm{Co})$, fluorspar $\left(\mathrm{CaF}_{2}\right)$, gallium $(\mathrm{Ga})$, germanium $(\mathrm{Ge})$, graphite $(\mathrm{C})$, hafnium $(\mathrm{Hf})$, helium $(\mathrm{He})$, indium (In), lithium (Li), magnesium (Mg), manganese (Mn), niobium $(\mathrm{Nb})$, platinum group elements (PGEs), potash $(\mathrm{KCl})$, rare earth elements (REE), rhenium (Re), rubidium $(\mathrm{Rb})$, scandium (Sc), strontium (Sr), tantalum (Ta), tellurium (Te), tin $(\mathrm{Sn})$, titanium (Ti), tungsten $(\mathrm{W})$, uranium $(\mathrm{U})$, vanadium (V), and zirconium (Zr).

In 2018, Congress allocated funds to the USGS Mineral Resources Program for the Earth Mapping Resources Initiative (Earth MRI), which is a partnership between the USGS, the Association of American State Geologists, and other Federal, State, and private-sector organizations. The goal of Earth MRI is to generate maps and data that aid in increasing the domestic inventory of critical minerals (Day, 2019). To reach this goal, focus areas with critical mineral resource potential must be defined and prioritized for new topographic, geologic, geochemical, and geophysical mapping; and funds must be allocated to States and contractors to conduct the work. The new maps of high priority focus areas are designed to (1) advance understanding of, or "image," the three-dimensional geologic framework, (2) stimulate exploration and development of domestic resources of critical minerals, and (3) decrease the Nation's reliance on foreign sources of critical minerals.

During Phase 1, focus areas with potential for REEbearing deposit types were targeted and classified by geologic environment (Dicken and others, 2019; Hammarstrom and Dicken, 2019). In 2019, funds were allocated to map the prioritized REE-focus areas and several studies were underway in 2020. During Phase 2, focus areas with potential for Al, C, Co, $\mathrm{Li}, \mathrm{Nb}, \mathrm{PGE}, \mathrm{Sn}, \mathrm{Ta}, \mathrm{Ti}$, and $\mathrm{W}$ were targeted and classified into mineral systems (explained in the "Mineral Systems" section) that generate ore deposits containing the aforementioned critical minerals. The plan for Phase 3 is to target and classify all or most of the remaining critical minerals $\left(\mathrm{As}, \mathrm{BaSO}_{4}, \mathrm{Be}\right.$, $\mathrm{Bi}, \mathrm{CaF}_{2}, \mathrm{Cr}, \mathrm{Cs}$, Ga, Ge, He, Hf, In, $\mathrm{KCl}, \mathrm{Mg}, \mathrm{Mn}, \mathrm{Re}, \mathrm{Rb}$, $\mathrm{Sb}, \mathrm{Sc}, \mathrm{Sr}, \mathrm{Te}, \mathrm{U}, \mathrm{V}$, and $\mathrm{Zr}$ ).

\section{Problem and Solution}

To define and prioritize Earth MRI focus areas across the United States for 35 critical minerals in a few years' time, an efficient method was needed that minimized the number of focus areas and the number of times that each focus area was considered. Application of the commodity-based approach utilized for REE in Phase 1 to the remaining 34 critical minerals would be redundant and inefficient because, unlike REE and 
a few other exceptions ( $\left.\mathrm{Al}, \mathrm{BaSO}_{4}, \mathrm{C}, \mathrm{PGE}\right)$, critical minerals generally do not constitute the major part of any single mineral deposit. Instead, they are more commonly present as minor constituents in deposits mined for principal commodities, such as gold $(\mathrm{Au})$, silver $(\mathrm{Ag})$, lead $(\mathrm{Pb})$, zinc $(\mathrm{Zn})$, copper $(\mathrm{Cu})$, molybdenum (Mo), nickel (Ni), iron (Fe), and phosphorus (P).

The solution to this problem, employed in Phases 2 and 3 of Earth MRI, was to take advantage of the hierarchical relationship that exists between mineral systems, ore deposits, principal commodities, and critical minerals (described in the "Table Rationale and Explanation" section) and define focus areas that correspond, more or less, to the footprint of mineral systems, and then prioritize each system-based focus area once for the entire suite of critical minerals that it may contain. An important advantage of this approach is that the scale of mineral systems is much larger than individual ore deposits and they generally have key geologic features that can be "imaged" by the Earth MRI mapping techniques described previously. The number of focus areas can be further reduced by prioritizing only the largest and most prospective systems in the United States because, in most (but not all) cases, small systems are unlikely to generate deposits that are large enough to contain significant quantities of critical minerals. Another way to minimize the number of focus areas is to group mineral systems that occur in clusters or belts into one focus area. Similarly, because well-endowed mineral systems are known to form in specific tectonic settings and during specific time periods of Earth history, such settings that have been identified in frontier areas or under cover can be designated as focus areas.

In the following sections, we describe mineral systems and the rationale for, and structure of, the systems, deposits, commodities, and critical minerals information compiled in table 1 (PDF file) and show how it can be used to streamline workflow for Earth MRI.

\section{Mineral Systems}

The mineral systems concept is based on current understanding of how ore deposits form and relate to broader geologic frameworks and the tectonic history of the Earth (for example, Wyborn and others, 1994; McCuaig and others, 2010; Huston and others, 2016; and Geological Survey of Western Australia, 2019). Mineral systems encompass all of the components required to form ore deposits (fig. 1). These components are (1) an optimum geotectonic setting, (2) energy to drive the system (heat, gravity), (3) source rocks for ligands and metals (igneous, metamorphic, or sedimentary rocks; preexisting mineralization), (4) a transport medium (melts, aqueous fluids-liquids-vapors, petroleum-natural gas), (5) transport pathways (channels, permeable structures and lithologies), (6) chemical and physical traps that concentrate metals to ore grades (deposits), and (7) distal expressions (mineral, chemical, or thermal anomalies) that extend to the limit of the system. In a given geotectonic setting, variations in these components, produce mineral systems and ore deposits of different types that are enriched in different principal commodities and byproducts, of which some are critical minerals.

Mineral systems with genetically related ore deposits generally form during an episode of magmatism, metamorphism, deformation, sedimentation, weathering, or erosion in specific geotectonic settings (fig. 1). The geotectonic setting includes the actual tectonic configuration as well as aspects of crustal evolution and (or) climatic conditions that are required for a system to produce significant deposits. If a setting lacks one or more key ingredients, such as dilatant structures, enriched source rocks, an arid climate, or appropriate physical or chemical conditions, a mineral system may operate without producing significant ore deposits. Systems generally require a trigger to get them started. Triggers can be sudden, such as volcanism above a mantle plume (for example, Ni-Cu-PGE deposits in a mafic magmatic system), or barely noticeable, such as formation of a peneplain in a tropical climatic zone (for example, bauxite deposits in a chemical weathering system).

The vertical and lateral extents of mineral systems are quite variable. For example, a system may have large vertical extents, as in porphyry Cu-Mo-Au systems that extend from the subduction zone to the surface (fig. $2 \mathrm{C}$ ), or short vertical extents, such as chemical weathering systems that are restricted to the vadose zone between the surface and the water table. Mineral systems can have large lateral extents, as in basin brine path systems that extend from marine evaporite basins, across passive margins, to shelf-slope breaks where they discharge into the ocean (fig. 3). Other mineral systems can have small lateral extents, such as in carbonatites. Most systems are spatially zoned such that deposits with different commodities and critical minerals occur at different levels or in proximal to distal positions (for example, figs. $2 A$ and 3 ). In some systems, critical minerals are enriched on the periphery of the system or deposit types within it, or they occur in unconventional deposit types (for example, alunite altered lithocaps). Some deposit types are mined for a single commodity, such as tungsten skarn deposits, whereas others are mined for several commodities, such as placer deposits mined for $\mathrm{Au}, \mathrm{REE}, \mathrm{Ti}$, and $\mathrm{Zr}$-Hf. In some deposit types, the principal commodity is a critical mineral, but in most cases critical minerals have been, or may only be, produced as byproducts of principal commodity deposits (Hayes and McCullough, 2018), such as REE from sedimentary phosphate deposits.

Detailed information on each system and deposit type is provided in the references cited in table 1.

\section{Table Rationale and Explanation}

Table 1 was populated with principal commodity and critical mineral information gathered from ore deposit models published by the USGS, other government organizations, and scientific journals. This information was classified into mineral systems using the concept outlined in the "Mineral Systems" section. This classification consisted of grouping deposit types 


\section{Components}

\section{Energy Ligand Source Transport Trap Outflow}
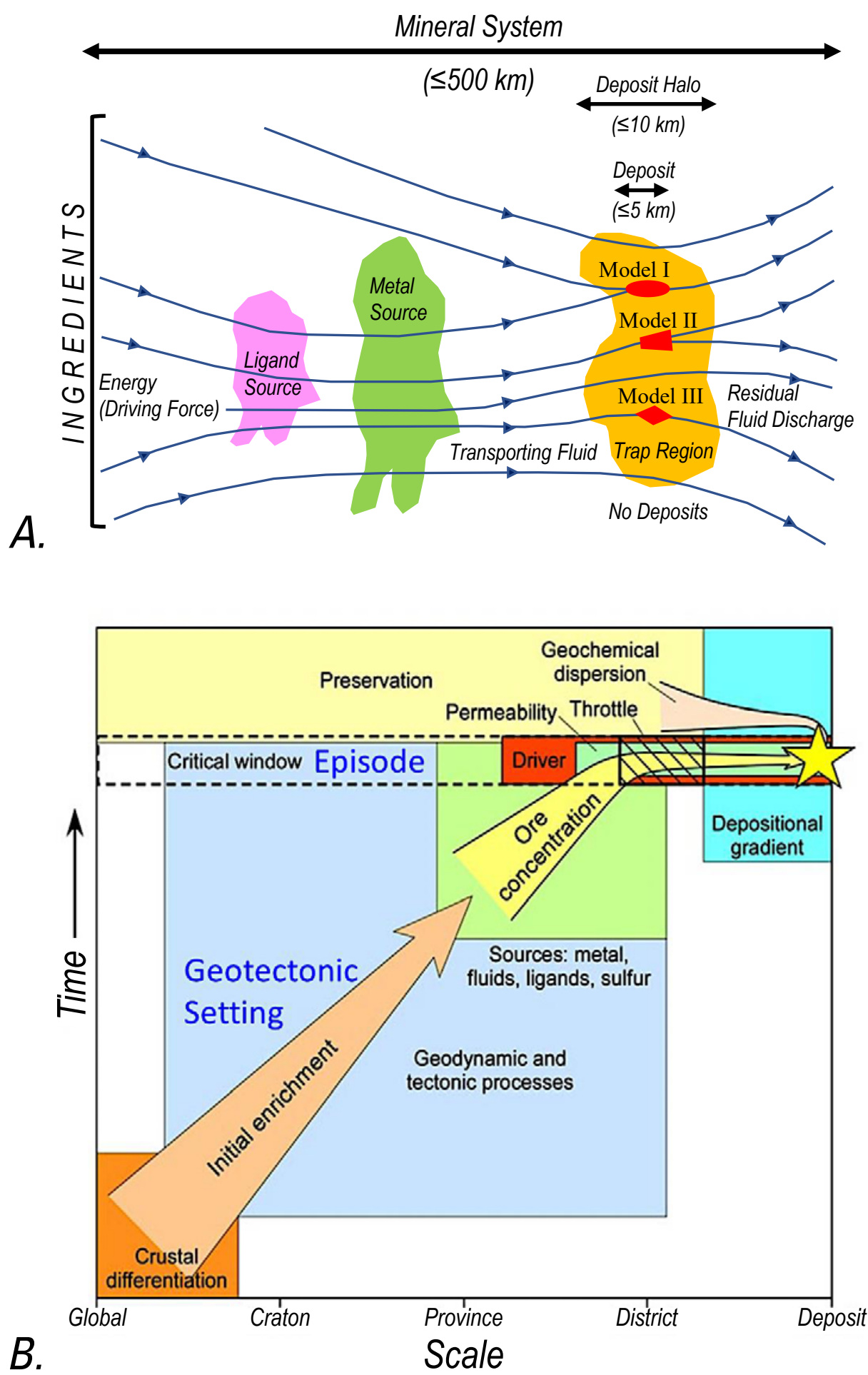

Figure 1. Mineral system concepts. A, Modified from Knox-Robinson and Wyborn (1997). $B$, Modified from Geoscience Australia (2019). ( $\leq$, less than or equal; km, kilometer) 

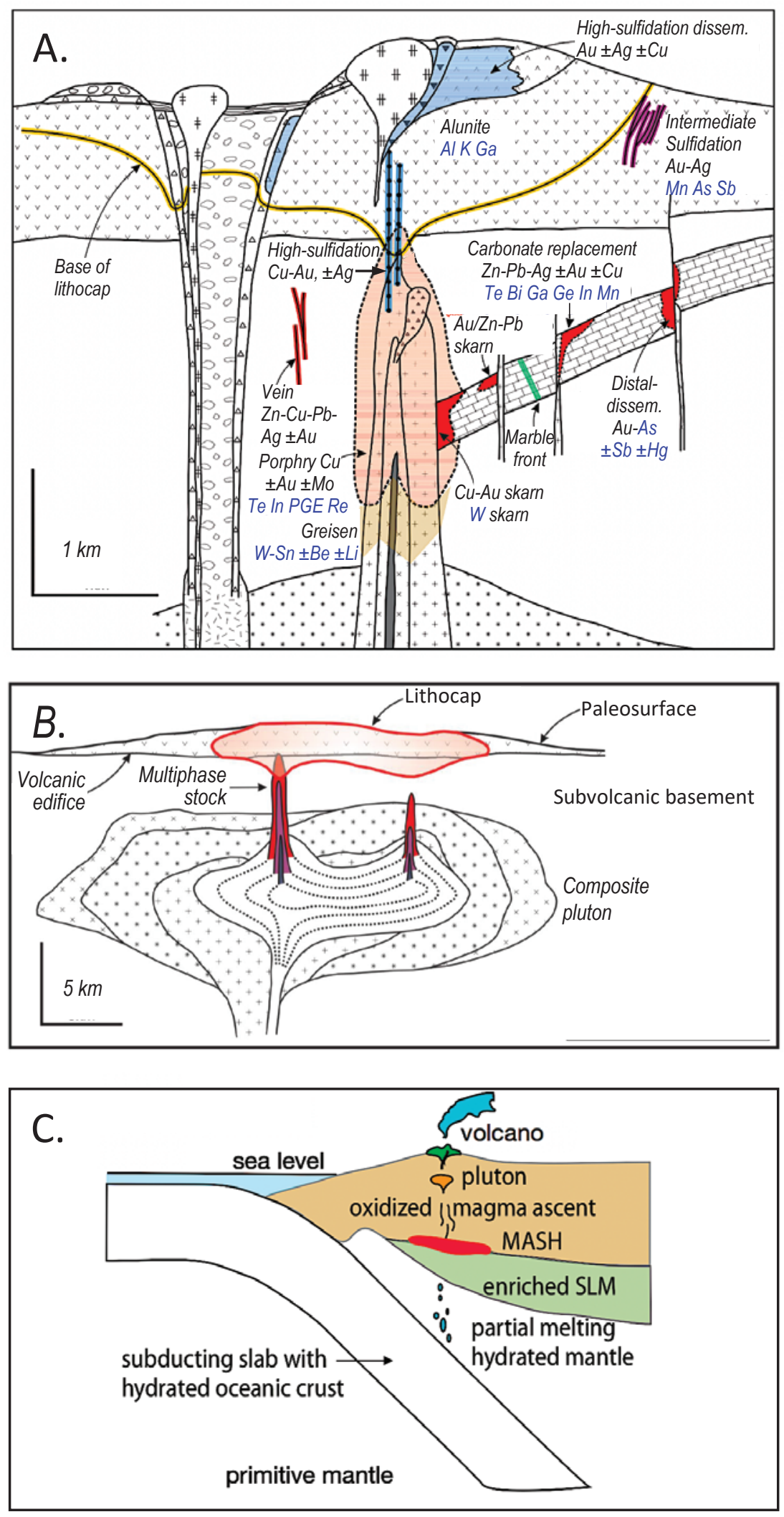

Figure 2. Schematic cross sections of a porphyry copper ( $\mathrm{Cu}$ )-molybdenum (Mo)-gold (Au) system (with critical minerals in blue) at various scales. $A$ and $B$, Modified from Sillitoe (2010). C, Modified from Tosdal and others (2009). (Ag, silver; Al, aluminum; As, arsenic; Be, beryllium; Bi, bismuth; Co, cobalt; dissem., disseminated; $\mathrm{Ga}$, gallium; $\mathrm{Hg}$, mercury; In, indium; $\mathrm{K}$, potassium; km, kilometer; Li, lithium; MASH, melting, assimilation, and homogenization; Mn, manganese; Pb, lead; PGE, platinum group elements; Re, rhenium; Sb, antimony; SLM, subcontinental lithospheric mantle; Sn, tin; Te, tellurium; W, tungsten; Zn, zinc) 


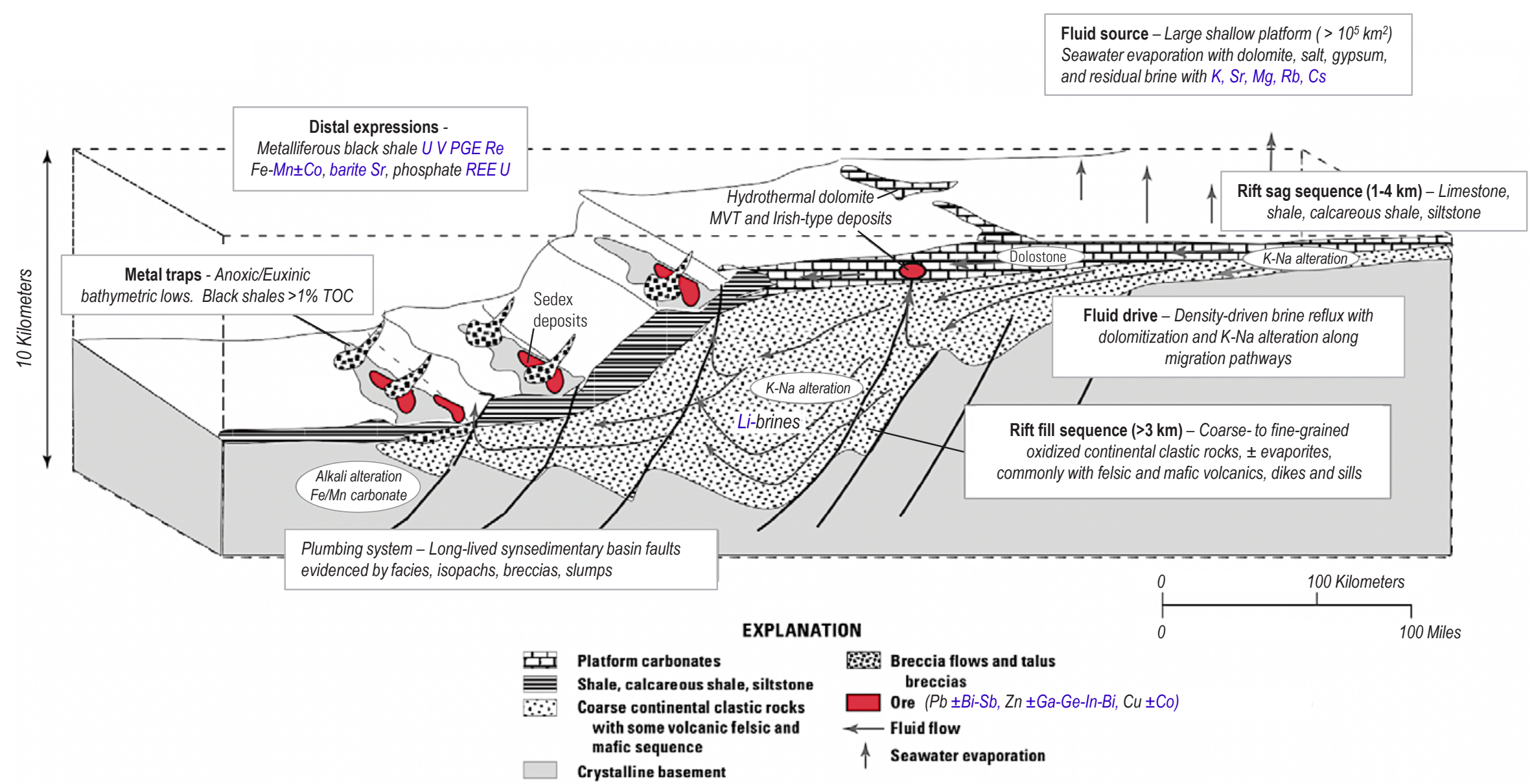

Figure 3. Schematic model of a basin brine path system (with critical minerals in blue), modified from Emsbo, 2009. (>, greater than; \%, percent; Ba, barium; Bi, bismuth; Co, cobalt; Cs, cesium; Cu, copper; Fe, iron; Ga, gallium; Ge, germanium; In, indium; K, potassium; km, kilometer; km², square kilometer; Li, lithium; Mg, magnesium; Mn, manganese; MVT, Mississippi Valley-type; $\mathrm{Na}$, sodium; $\mathrm{Pb}$, lead; $\mathrm{PGE}$, platinum group elements; $\mathrm{PO}_{4}$, phosphate; $\mathrm{Rb}$, rubidium; Re, rhenium; $\mathrm{REE}$, rare earth elements; $\mathrm{U}$, uranium; V, vanadium; Sb, antimony; Sr, strontium; TOC, total organic carbon; Zn, zinc) 
Systems-Deposits-Commodities-Critical Minerals Table for the Earth Mapping Resources Initiative

that share a fundamental genetic relationship to geologic controls that are characteristic of each system type. Thus, if one part (for example, deposit type) of a system is identified, then the other parts (for example, other deposit types) of the system may be present nearby.

As table 1 was being constructed, it became clear that it could be simplified and made more useful for Earth MRI by (1) grouping deposit types with similar mineral assemblages that contain similar element suites, and (or) (2) splitting out deposit types with distinct mineralogies and elements. This decision was based on the common mineral associations that occur in certain deposit types and the typical element substitutions that occur in each mineral. For example, in porphyry $\mathrm{Cu}-\mathrm{Mo}-\mathrm{Au}$ systems (fig. $2 A-B$ ), polymetallic skarn, replacement, vein, and intermediate sulfidation (SRVIS) deposits all contain various proportions of $\mathrm{Cu}-, \mathrm{Zn}$-, and $\mathrm{Pb}$-sulfides and As- and Sb-sulfosalts with variable proportions of the same principal commodities $(\mathrm{Cu}, \mathrm{Zn}, \mathrm{Pb}, \mathrm{Ag}, \mathrm{Au})$ and critical minerals ( $\mathrm{Ge}$, $\mathrm{Ga}, \mathrm{In}, \mathrm{Bi}, \mathrm{Sb}, \mathrm{As}, \mathrm{W}, \mathrm{Te})$. Thus, an overarching deposit name was devised to encompass them, "polymetallic SRVIS." In an analogous way, $\mathrm{Cu}$-sulfides in porphyry and skarn copper deposits typically contain PGE, Te, and Bi; molybdenite in porphyry and skarn molybdenum deposits contains Re; pyrite in distal disseminated silver-gold deposits contains $\mathrm{As}$ and $\mathrm{Sb}$; and alunite in lithocap deposits contains $\mathrm{Al}, \mathrm{K}$, and $\mathrm{Ga}$. Placers are more complex because the assemblage of ore minerals that they contain reflects the assemblage of source rocks and mineralization exposed in the catchment area. Consequently, it is important to understand that the distinctions made in table 1 are idealized and that in nature the deposit types grade into, or overlap with, one another. Nevertheless, the deposit groupings and distinctions can be used to identify the parts or aspects of a mineral system that are likely to be enriched in specific principal commodities and critical minerals.

\section{Table Structure}

The table consists of six columns (with headers in bold type). The first is the "System name." In some cases, an established name was used, for example, "Placer." In other cases, a name was selected that emphasizes an aspect of the system that is characteristic of, and distinct from, the other systems, for example, "Chemical Weathering." One system was named after the principal deposit type within it, namely "Porphyry $\mathrm{Cu}-\mathrm{Mo}-\mathrm{Au}$." In this case, it is important to realize that porphyry $\mathrm{Cu}-\mathrm{Mo}-\mathrm{Au}$ systems are much larger than porphyry $\mathrm{Cu}-\mathrm{Mo}-\mathrm{Au}$ deposits and encompass key aspects of the tectonic framework and all of the deposit types that occur within the system, as shown in figure 2. The second column is a brief "Synopsis" that provides information on the geotectonic setting of the system and a description of how it operates to form ore deposits containing various principal commodities and critical minerals. The third column is "Deposit types." As described in the previous section, in some cases, different deposit types were grouped together under an overarching deposit name because they contain a similar assortment of principal commodities and critical minerals whereas those with distinct principal commodities and critical minerals were split out. The fourth column is a list of "Principal commodities" that generally are produced from, or explored for, in the deposit type. These are the commodities that govern the economics of mining and mineral processing. The fifth column is a list of "Critical minerals." Those that have actually been produced from the deposit type are highlighted in bold type (for example, REE), whereas those that are enriched in the deposit type, but have not yet been produced, are listed in italics (for example, $P G E$ ). Critical minerals that are principal commodities, are listed in both columns. The sixth column is "Reference(s)," which cites publications that contain detailed descriptions of the system and deposit types upon which the entries in table 1 are based.

\section{Table Use}

The hierarchical relationship between systems, deposits, commodities, and critical minerals in table 1 can be used to help define and prioritize Earth MRI focus areas for mapping projects in four ways.

First, if any part of a mineral system has been recognized by previous work, table 1 can be used to deduce the assortment of deposit types, principal commodities, and critical minerals that may be present in adjacent areas and under cover. Because information generally exists on the principal commodities and deposit types that are present in well-explored areas with a history of mining, table 1 can be used to infer the system type(s) and the critical minerals that may be present in mine waste, unmined resources, concealed deposit types under cover, or in deposit types that were removed by erosion. In areas with historical mining and exploration, these inferences have a higher degree of certainty because the known deposit types confirm that a mineral system actually operated in the area. The deposit types recognized at or near the surface also provide an indication of the level of exposure or tilting of the system.

Second, for system-based focus areas of the same type (for example, porphry $\mathrm{Cu}-\mathrm{Mo}-\mathrm{Au}$ ), the attributes of each area can be compared to identify those that are well endowed and (or) would benefit the most from Earth MRI mapping techniques.

Third, in some parts of the country, systems of different types and ages occur in the same geographic area, such that the system-based focused areas overlap. These areas are highly prospective and may benefit the most from Earth MRI mapping efforts.

Fourth, in frontier areas (for example, Alaska) or areas with extensive cover (for example, U.S. mid-continent), if a geotectonic setting, or terrane, is recognized that is known to host mineral systems of a given type elsewhere in the world (for example, Mesoproterozoic magmatic provinces), table 1 can be used to infer the deposit types, principal commodities, and critical minerals that may be present. In this case, Earth MRI maps of such terranes may detect evidence of mineral systems and lead to new discoveries. 
Table 1. Systems-Deposits-Commodities-Critical Minerals Table for the Earth Mapping Resources Initiative.

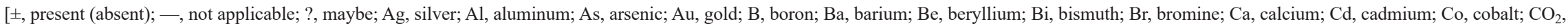
carbon dioxide; Cs, cesium; Cr, chromium; Cu, copper; F, fluorine; Fe, iron; Ga, gallium; Ge, germanium; Hf, hafnium; Hg, mercury; I, iodine; IAEA, International Atomic Energy Agency; In, indium; IOA, iron

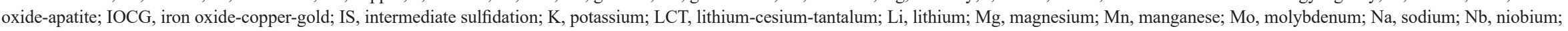
$\mathrm{Ni}$, nickel; NYF, niobium-yttrium-fluorine; P, phosphorus; Pb, lead; PGE, platinum group elements; R, replacement; Rb, rubidium; Re, rhenium; REE, rare earth elements; S, skarn; Sb, antimony; Sc, scandium; Se,

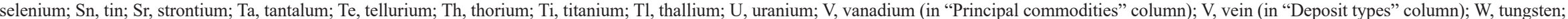
$\mathrm{Y}$, yttrium; Zn, zinc; Zr, zirconium]

\begin{tabular}{|c|c|c|c|c|c|}
\hline System name & Synopsis & Deposit types & Principal commodities & Critical minerals ${ }^{1}$ & Reference(s) \\
\hline \multirow{15}{*}{$\begin{array}{l}\text { Placer (riverine-marine, } \\
\text { residual-eluvial-alluvial- } \\
\text { shoreline, paleo) }\end{array}$} & \multirow{15}{*}{$\begin{array}{l}\text { Placer systems operate in drainage } \\
\text { basins and along shorelines where } \\
\text { there is either topographic relief and } \\
\text { gravity-driven turbulent flow of sur- } \\
\text { face water or tidal and wind-driven } \\
\text { wave action. Placer systems con- } \\
\text { centrate insoluble resistate minerals } \\
\text { liberated from various rock types } \\
\text { and mineral occurrences by the } \\
\text { chemical breakdown and winnowing } \\
\text { away of enclosing minerals by the } \\
\text { movement of water. The distribu- } \\
\text { tion of insoluble resistate minerals is } \\
\text { controlled by their size, density, and } \\
\text { the turbulence of fluid flow. }\end{array}$} & Gold & $\mathrm{Au}$ & - & \multirow{15}{*}{$\begin{array}{l}\text { Sloan, 1964; Levson, } \\
\text { 1995; Van Gosen } \\
\text { and others, 2014; } \\
\text { Sengupta and } \\
\text { Van Gosen, 2016; } \\
\text { Jones and others, } \\
\text { 2017; Wang and } \\
\text { others, } 2021\end{array}$} \\
\hline & & Uraninite, autunite-group minerals & $\mathrm{U}$ & $\mathbf{U}$ & \\
\hline & & PGE & PGE & PGE & \\
\hline & & Cassiterite & $\mathrm{Sn}$ & Sn, Sc & \\
\hline & & Wolframite/scheelite & $\mathrm{W}$ & $\mathbf{W}, S c$ & \\
\hline & & Barite & Barite & Barite & \\
\hline & & Fluorite & Fluorite & Fluorite & \\
\hline & & Monazite/xenotime & REE, Y, Th & REE & \\
\hline & & Columbite/tantalite & $\mathrm{Nb}, \mathrm{Ta}$ & Nb, Ta, $M n$ & \\
\hline & & Zircon & $\mathrm{Zr}, \mathrm{Hf}$ & $\mathrm{Zr}, \mathrm{Hf}$ & \\
\hline & & Ilmenite/rutile/leucoxene & $\mathrm{Ti}$ & Ti, $S c$ & \\
\hline & & Magnetite/hematite/goethite & $\mathrm{Fe}$ & - & \\
\hline & & Diamond & Diamond gems and abrasive & - & \\
\hline & & Sapphire & Sapphire gems & - & \\
\hline & & Garnet & Garnet gems and abrasive & - & \\
\hline
\end{tabular}


[ \pm , present (absent); - , not applicable; ?, maybe; Ag, silver; Al, aluminum; As, arsenic; Au, gold; B, boron; $\mathrm{Ba}$, barium; $\mathrm{Be}$, beryllium; $\mathrm{Bi}$, bismuth; $\mathrm{Br}$, bromine; $\mathrm{Ca}$, calcium; $\mathrm{Cd}$, cadmium; Co, cobalt; $\mathrm{CO}$, carbon dioxide; Cs, cesium; Cr, chromium; Cu, copper; F, fluorine; Fe, iron; Ga, gallium; Ge, germanium; Hf, hafnium; Hg, mercury; I, iodine; IAEA, International Atomic Energy Agency; In, indium; IOA, iron

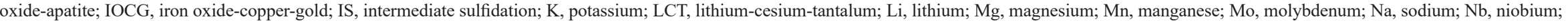

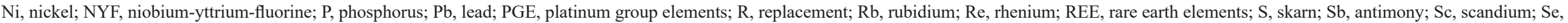

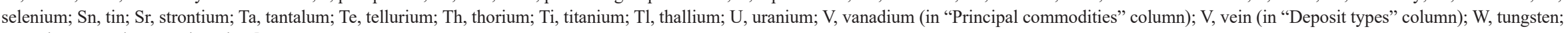
Y, yttrium; Zn, zinc; Zr, zirconium]

\begin{tabular}{|c|c|c|c|c|c|}
\hline System name & Synopsis & Deposit types & Principal commodities & Critical minerals 1 & Reference(s) \\
\hline \multirow{18}{*}{$\begin{array}{l}\text { Chemical weathering } \\
\text { (unsaturated zone, in situ) }\end{array}$} & \multirow{18}{*}{$\begin{array}{l}\text { Chemical weathering systems operate in } \\
\text { stable areas of low to moderate relief } \\
\text { with sufficient rainfall to chemically } \\
\text { dissolve and concentrate elements } \\
\text { present in various rock types and } \\
\text { mineral occurrences by the down- } \\
\text { ward percolation of surface water } \\
\text { in the unsaturated zone. Chemical } \\
\text { gradients cause different elements to } \\
\text { be concentrated at different positions } \\
\text { in the weathering profile and at the } \\
\text { water table. Bauxite, Ni-laterite, and } \\
\text { carbonatite laterite are restricted } \\
\text { to tropical climatic zones; others } \\
\text { form in temperate and arid climates. } \\
\text { Dissolved uranium is reduced on } \\
\text { carbonaceous material in lakes and } \\
\text { swamps. Dissolved manganese pre- } \\
\text { ciptates at redox interfaces in lakes. }\end{array}$} & Nickel-cobalt laterite & $\mathrm{Ni}, \mathrm{Co}$ & Co, $M n, S c$ & \multirow{18}{*}{$\begin{array}{l}\text { Otton and others, } \\
\text { 1990; Long and } \\
\text { others, 1992; } \\
\text { Marsh and others, } \\
\text { 2013; Foley and } \\
\text { Ayuso, 2015; Bru- } \\
\text { neton and Cuney, } \\
\text { 2016; Sanematsu } \\
\text { and Watanabe, } \\
\text { 2016; IAEA, } \\
\text { 2020; Wang and } \\
\text { others, 2021 }\end{array}$} \\
\hline & & Bauxite & $\mathrm{Al}$ & Al, $G a, R E E$ & \\
\hline & & Clay & Kaolin & $G a, L i, R E E$ & \\
\hline & & Carbonatite laterite & $\mathrm{Nb}, \mathrm{REE}$ & Nb, REE, $S c$ & \\
\hline & & Regolith (Ion adsorption) REE & REE & REE, $S c$ & \\
\hline & & Surficial uranium & $\mathrm{U}$ & $\mathbf{U}$ & \\
\hline & & Lacustrine carbonaceous uranium & $\mathrm{U}$ & $\mathbf{U}$ & \\
\hline & & Coal uranium & $\mathrm{U}$ & $\mathbf{U}$ & \\
\hline & & Lacustrine manganese & $\mathrm{Mn}$ & Mn & \\
\hline & & Supergene (and laterite) gold & $\mathrm{Au}$ & - & \\
\hline & & Supergene silver & $\mathrm{Ag}$ & $?$ & \\
\hline & & Supergene lead & $\mathrm{Pb}$ & $?$ & \\
\hline & & Supergene zinc & $\mathrm{Zn}$ & ?Ge, Ga, In? & \\
\hline & & Supergene (and exotic) copper & $\mathrm{Cu}$ & ?Te, Bi? & \\
\hline & & Supergene cobalt & Co & Co & \\
\hline & & Supergene PGE & PGE & PGE & \\
\hline & & Supergene manganese & $\mathrm{Mn}$ & $\mathrm{Mn}, \mathrm{Co}$ & \\
\hline & & Supergene iron & $\mathrm{Fe}$ & $M n$ & \\
\hline
\end{tabular}


Table 1. Systems-Deposits-Commodities-Critical Minerals Table for the Earth Mapping Resources Initiative.-Continued

[ \pm , present (absent); - , not applicable; ?, maybe; $\mathrm{Ag}$, silver; $\mathrm{Al}$, aluminum; $\mathrm{As}$, arsenic; $\mathrm{Au}$, gold; $\mathrm{B}$, boron; $\mathrm{Ba}$, barium; $\mathrm{Be}$, beryllium; $\mathrm{Bi}$, bismuth; $\mathrm{Br}$, bromine; $\mathrm{Ca}$, calcium; $\mathrm{Cd}$, cadmium; $\mathrm{Co}$, cobalt; $\mathrm{CO}$ 2, carbon dioxide; Cs, cesium; Cr, chromium; Cu, copper; F, fluorine; Fe, iron; Ga, gallium; Ge, germanium; Hf, hafnium; Hg, mercury; I, iodine; IAEA, International Atomic Energy Agency; In, indium; IOA, iron

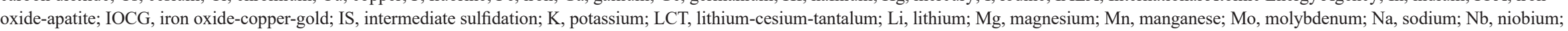

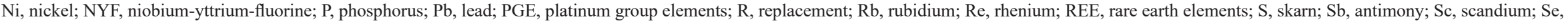
selenium; Sn, tin; Sr, strontium; Ta, tantalum; Te, tellurium; Th, thorium; Ti, titanium; Tl, thallium; U, uranium; V, vanadium (in "Principal commodities" column); V, vein (in "Deposit types" column); W, tungsten; $\mathrm{Y}$, yttrium; Zn, zinc; Zr, zirconium]

\begin{tabular}{|c|c|c|c|c|c|}
\hline System name & Synopsis & Deposit types & Principal commodities & Critical minerals 1 & Reference(s) \\
\hline \multirow[t]{5}{*}{ Meteoric recharge } & \multirow{5}{*}{$\begin{array}{l}\text { Meteoric recharge systems operate } \\
\text { where oxidized meteoric ground- } \\
\text { water displaces reduced connate } \\
\text { water in sandstone aquifers that } \\
\text { often contain volcanic ash or where } \\
\text { such groundwater evaporates at the } \\
\text { surface. As oxidized water de- } \\
\text { scends through sandstone aquifers, } \\
\text { it scavenges uranium and other } \\
\text { elements from detrital minerals and } \\
\text { (or) volcanic glass. Uranium and } \\
\text { other elements precipitate at the } \\
\text { redox front with reduced connate } \\
\text { water, on carbonaceous material } \\
\text { in the aquifers, or at the surface in } \\
\text { calcrete by evaporation. In gran- } \\
\text { ite, descending oxidized meteoric } \\
\text { groundwater leaches uranium from } \\
\text { zircon, apatite, and other minerals } \\
\text { that precipitate by reactions with } \\
\text { ferrous Fe minerals. In ultramafic } \\
\text { rocks, dissolved CO }{ }_{2} \text { in descending } \\
\text { meteoric groundwater reacts with } \\
\text { Mg-silicates to form magnesite, } \\
\text { which may also precipitate in per- } \\
\text { meable sediment or rocks nearby. }\end{array}$} & Sandstone uranium & $\mathrm{U}, \mathrm{V}$ & $\begin{array}{l}\mathbf{U}, \mathbf{V}, \mathbf{R e}, \mathbf{S c}, \mathbf{R E E}, \\
\mathrm{Mn}, C o, P G E\end{array}$ & \multirow{5}{*}{$\begin{array}{l}\text { Zachmann and } \\
\text { Johannes, 1989; } \\
\text { Skirrow and } \\
\text { others, 2009; } \\
\text { Breit, 2016; } \\
\text { Bruneton and } \\
\text { Cuney, 2016; Hall } \\
\text { and others, 2019; } \\
\text { IAEA, 2020 }\end{array}$} \\
\hline & & Carbonate uranium & U, V & $\begin{array}{l}\text { U, V, Re, Sc, REE, } \\
\quad C o, P G E\end{array}$ & \\
\hline & & Calcrete uranium & U, V & $\mathbf{U}, \mathbf{V}, S r$ & \\
\hline & & Granite uranium & $\mathrm{U}$ & $\mathbf{U}$ & \\
\hline & & Cryptocrystalline magnesite & $\mathrm{Mg}$ & Mg & \\
\hline \multirow[t]{3}{*}{ Meteoric convection } & \multirow{3}{*}{$\begin{array}{l}\text { Low-sulfidation Au-Ag deposits as- } \\
\text { sociated with mantle plume volcanic } \\
\text { rocks form under relatively low oxy- } \\
\text { gen and sulfur fugacities, and have } \\
\text { low base metal contents and high } \\
\text { Au/Ag ratios and selenium contents. } \\
\text { Low-sulfidation deposits also occur } \\
\text { along extensional fault zones that } \\
\text { are not associated with proximal, } \\
\text { coeval magmatic activity. }\end{array}$} & $\begin{array}{l}\text { Low sulfidation epithermal } \\
\text { gold-silver }\end{array}$ & $\mathrm{Au}, \mathrm{Ag}, \mathrm{Hg}, \mathrm{Sb}$ & $\mathbf{S b}, T e$ & \multirow[t]{3}{*}{$\begin{array}{l}\text { Simmons and others, } \\
\text { 2005; John and } \\
\text { Henry, } 2020\end{array}$} \\
\hline & & $\begin{array}{l}\text { Low sulfidation epithermal } \\
\text { antimony }\end{array}$ & $\mathrm{Sb}$ & $\mathbf{S b}$ & \\
\hline & & $\begin{array}{l}\text { Low sulfidation epithermal } \\
\text { mercury }\end{array}$ & $\mathrm{Hg}$ & - & \\
\hline
\end{tabular}


[ \pm , present (absent); - , not applicable; ?, maybe; Ag, silver; Al, aluminum; As, arsenic; Au, gold; B, boron; Ba, barium; $\mathrm{Be}$, beryllium; $\mathrm{Bi}$, bismuth; $\mathrm{Br}$, bromine; $\mathrm{Ca}$, calcium; $\mathrm{Cd}$, cadmium; $\mathrm{Co}$, cobalt; $\mathrm{CO}$, carbon dioxide; Cs, cesium; Cr, chromium; Cu, copper; F, fluorine; Fe, iron; Ga, gallium; Ge, germanium; Hf, hafnium; Hg, mercury; I, iodine; IAEA, International Atomic Energy Agency; In, indium; IOA, iron

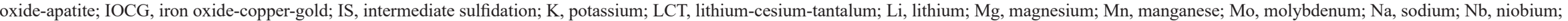

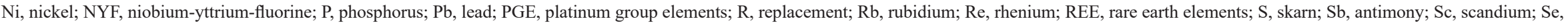

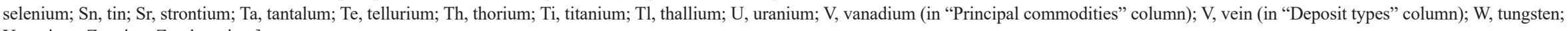
Y, yttrium; Zn, zinc; Zr, zirconium]

\begin{tabular}{|c|c|c|c|c|c|}
\hline System name & Synopsis & Deposit types & Principal commodities & Critical minerals 1 & Reference(s) \\
\hline \multirow[t]{11}{*}{ Lacustrine evaporite } & \multirow{11}{*}{$\begin{array}{l}\text { Lacustrine evaporite systems operate } \\
\text { in closed drainage basins in arid to } \\
\text { hyperarid climatic zones. Elements } \\
\text { present in meteoric surface, ground, } \\
\text { and geothermal recharge water are } \\
\text { concentrated by evaporation. As } \\
\text { salinity increases, evaporite minerals } \\
\text { typically precipitate in the follow- } \\
\text { ing sequence: gypsum or anhydrite, } \\
\text { halite, sylvite, carnallite, borate. } \\
\text { Nitrates are concentrated in basins } \\
\text { that accumulate sea spray. Residual } \\
\text { brines enriched in lithium and } \\
\text { other elements often accumulate in } \\
\text { aquifers below dry lake beds. Li-clay } \\
\text { and Li-B-zeolite deposits form where } \\
\text { residual brine reacts with lake sedi- } \\
\text { ment, ash layers, or volcanic rocks. }\end{array}$} & Trona & Soda ash $\left(\mathrm{Na}_{2} \mathrm{CO}_{3}\right)$ & - & \multirow{11}{*}{$\begin{array}{l}\text { Dyni, 1991; } \\
\text { Sheppard, } \\
\text { 1991a,b; Williams- } \\
\text { Stroud, 1991; } \\
\text { Orris, 1995; } \\
\text { Warren, 2010; } \\
\text { Bradley and } \\
\text { others, 2013; } \\
\text { Hofstra and } \\
\text { others, 2013b; } \\
\text { Munk and others, } \\
\text { 2016; Bradley and } \\
\text { others, 2017b; } \\
\text { Power and others, } \\
2019\end{array}$} \\
\hline & & Gypsum & Gypsum $\left(\mathrm{CaSO}_{4} \cdot 2 \mathrm{H}_{2} \mathrm{O}\right)$ & - & \\
\hline & & Salt & Salt $(\mathrm{NaCl})$ & - & \\
\hline & & Potash & Potash $(\mathrm{KCl})$ & Potash & \\
\hline & & Carnallite & Carnellite $\left(\mathrm{KMgCl}_{3} \bullet 6 \mathrm{H}_{2} \mathrm{O}\right)$ & Potash, Mg & \\
\hline & & Magnesite & $\mathrm{MgCO}_{3}$ & Mg & \\
\hline & & Borate & Borax, boric Acid & $\mathrm{Li}$ & \\
\hline & & Nitrate & $\begin{array}{c}{[\mathrm{Na}, \mathrm{K}, \mathrm{Ca}, \mathrm{Mg}]\left[\mathrm{NO}_{3} \text { nitrate }\right.} \\
\left.\mathrm{IO}_{3} \text { iodate, } \mathrm{BO}_{3} \text { borate }\right]\end{array}$ & $M g$ & \\
\hline & & Residual brine & $\begin{array}{l}\text { Salt, potash, borax, boric acid, } \\
\text { soda ash, sodium sulfate, Li, } \\
\text { Rb, Cs, Mg, Mn, Sr, Br, I, } \\
\text { W, Zn }\end{array}$ & $\begin{array}{l}\text { Potash, Li, } M n, \\
\quad R b, C s, M g \\
\quad S r, W\end{array}$ & \\
\hline & & Lithium clay & $\mathrm{Li}$ & $\mathbf{L i}$ & \\
\hline & & Lithium-boron zeolite & Zeolite, B, Li & $\mathrm{Li}$ & \\
\hline \multirow[t]{6}{*}{ Marine evaporite } & \multirow{6}{*}{$\begin{array}{l}\text { Marine evaporite systems operate in } \\
\text { shallow restricted epicontinental } \\
\text { basins in arid to hyperarid climatic } \\
\text { zones. Sabkha dolomite and sedimen- } \\
\text { tary magnesite form in coastal salt } \\
\text { flats and lagoons. Elements present in } \\
\text { seawater are concentrated by evapo- } \\
\text { ration. As salinity increases, evaporite } \\
\text { minerals typically precipitate in } \\
\text { the following sequence: gypsum or } \\
\text { anhydrite, halite, sylvite. Residual } \\
\text { basin brines are enriched in con- } \\
\text { served elements, such as Mg and Li. } \\
\text { Incursion of freshwater or seawater } \\
\text { can produce halite dissolution brines. }\end{array}$} & Sabkha dolomite & Building stone, aggregate, $\mathrm{Mg}$ & Mg & \multirow{6}{*}{$\begin{array}{l}\text { Raup 1991a, b; } \\
\text { Mountney, 2005; } \\
\text { Warren, 2010; } \\
\text { Horn and others, } \\
2017\end{array}$} \\
\hline & & Sedimentary magnesite & $\mathrm{Mg}$ & Mg & \\
\hline & & Gypsum & Gypsum $\left(\mathrm{CaSO}_{4} \cdot 2 \mathrm{H}_{2} \mathrm{O}\right)$ & - & \\
\hline & & Salt & Salt $(\mathrm{NaCl})$ & - & \\
\hline & & Potash & Potash $(\mathrm{KCl})$ & Potash & \\
\hline & & Dissolution brine & Petroleum, salt $(\mathrm{NaCl})$ & - & \\
\hline
\end{tabular}


Table 1. Systems-Deposits-Commodities-Critical Minerals Table for the Earth Mapping Resources Initiative.-Continued

[ \pm , present (absent); - , not applicable; ?, maybe; $\mathrm{Ag}$, silver; $\mathrm{Al}$, aluminum; $\mathrm{As}$, arsenic; $\mathrm{Au}$, gold; $\mathrm{B}$, boron; $\mathrm{Ba}$, barium; $\mathrm{Be}$, beryllium; $\mathrm{Bi}$, bismuth; $\mathrm{Br}$, bromine; $\mathrm{Ca}$, calcium; $\mathrm{Cd}$, cadmium; $\mathrm{Co}$, cobalt; $\mathrm{CO}$, carbon dioxide; Cs, cesium; Cr, chromium; Cu, copper; F, fluorine; Fe, iron; Ga, gallium; Ge, germanium; Hf, hafnium; Hg, mercury; I, iodine; IAEA, International Atomic Energy Agency; In, indium; IOA, iron

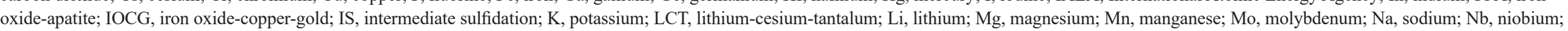

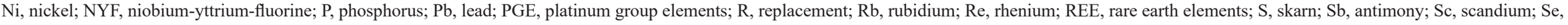
selenium; Sn, tin; Sr, strontium; Ta, tantalum; Te, tellurium; Th, thorium; Ti, titanium; Tl, thallium; U, uranium; V, vanadium (in "Principal commodities" column); V, vein (in "Deposit types" column); W, tungsten; Y, yttrium; Zn, zinc; Zr, zirconium]

\begin{tabular}{|c|c|c|c|c|c|}
\hline System name & Synopsis & Deposit types & Principal commodities & Critical minerals' 1 & Reference(s) \\
\hline \multirow[t]{7}{*}{ Basin brine path } & \multirow{7}{*}{$\begin{array}{l}\text { Basin brine path systems emanate from } \\
\text { marine evaporite basins and extend } \\
\text { downward and laterally through per- } \\
\text { meable strata to discharge points in } \\
\text { the ocean. Limestone is replaced by } \\
\text { reflux dolomite at low temperatures } \\
\text { and hydrothermal dolomite at high } \\
\text { temperatures. Basin brines evolve } \\
\text { to become ore fluids by scavenging } \\
\text { metals from various rock types along } \\
\text { gravity-driven flow paths. The min- } \\
\text { eralogy of the aquifers controls the re- } \\
\text { dox and sulfidation state of the brine } \\
\text { and the suite of elements that can be } \\
\text { scavenged. Cu- and Pb-Zn sulfide } \\
\text { deposits form where oxidized brines } \\
\text { encounter reduced S. Unconformity } \\
\text { U deposits form where oxidized } \\
\text { brines are reduced. Ba and Sr deposits } \\
\text { form where reduced brines encounter } \\
\text { marine sulfate or carbonate. }\end{array}$} & Basin brine & $\begin{array}{l}\text { Petroleum, salt, potash, Li, Rb, } \\
\quad \mathrm{Cs}, \mathrm{Mg}, \mathrm{Sr}, \mathrm{Br}, \mathrm{I}, \mathrm{Zn}\end{array}$ & $\begin{array}{l}\text { Potash, } L i, R b, C s \\
\quad M g, S r\end{array}$ & \multirow{7}{*}{$\begin{array}{l}\text { Cox and Singer, } \\
\text { 2007; Skirrow } \\
\text { and others, 2009; } \\
\text { Alpine, 2010; } \\
\text { Leach and others, } \\
\text { 2010; Hayes and } \\
\text { others, 2015; } \\
\text { Emsbo and others, } \\
\text { 2016a; Marsh } \\
\text { and others, 2016; } \\
\text { Johnson and } \\
\text { others, 2017; } \\
\text { Manning and } \\
\text { Emsbo, 2018 }\end{array}$} \\
\hline & & Reflux and hydrothermal dolomite & Building stone, aggregate, $\mathrm{Mg}$ & Mg & \\
\hline & & Zinc-lead (MVT and sedex) & $\mathrm{Zn}, \mathrm{Pb}, \mathrm{Ag}, \mathrm{Cu}, \mathrm{Co}$ & Sn, Ge, Co, $G a, I n$ & \\
\hline & & $\begin{array}{l}\text { Copper (sed-hosted and replace- } \\
\text { ment) }\end{array}$ & $\mathrm{Cu}, \mathrm{Co}, \mathrm{Ag}, \mathrm{Pb}, \mathrm{Zn}$ & $\begin{array}{l}\text { Co, PGE, Re, Ge, } \\
\text { Ga, } V, U \\
\end{array}$ & \\
\hline & & $\begin{array}{l}\text { Uranium (unconformity and brec- } \\
\text { cia pipe) }\end{array}$ & $\begin{array}{l}\text { U, V, Cu, Co, Mo, Re, Se, Sc, } \\
\text { REE }\end{array}$ & $\begin{array}{l}\text { U, V, Re, Sc, REE, } \\
\quad C o\end{array}$ & \\
\hline & & Barite (replacement and bedded) & Barite (witherite) & Barite & \\
\hline & & $\begin{array}{l}\text { Strontium (replacement and } \\
\text { bedded) }\end{array}$ & $\mathrm{Sr}$ (celestite, strontianite) & $\mathrm{Sr}$ & \\
\hline \multirow[t]{4}{*}{$\begin{array}{l}\text { Marine chemocline (bath- } \\
\text { tub rim) }\end{array}$} & \multirow{4}{*}{$\begin{array}{l}\text { Marine chemocline systems operate } \\
\text { where basin brines discharge into } \\
\text { the ocean. Consequent increases in } \\
\text { bioproductivity produce metallifer- } \\
\text { ous black shales. Changes in ocean } \\
\text { chemistry (oceanic anoxic events) } \\
\text { and development of chemoclines } \\
\text { result in chemical sedimentation of } \\
\text { phosphate and Mn and Fe carbon- } \\
\text { ates and oxides. }\end{array}$} & Black shale & $\begin{array}{l}\text { Stone coal, petroleum, } \mathrm{V}, \mathrm{Ni}, \\
\mathrm{Mo}, \mathrm{Au}, \mathrm{PGE}\end{array}$ & $\mathbf{V}, \operatorname{Re}, P G E, C r, U$ & \multirow{4}{*}{$\begin{array}{l}\text { Lefebure and } \\
\text { Coveney, 1995; } \\
\text { Force and others, } \\
\text { 1999; Emsbo, } \\
\text { 2000; Emsbo } \\
\text { and others, 2015, } \\
\text { 2016b; Cannon } \\
\text { and others, } 2017\end{array}$} \\
\hline & & Phosphate & Phosphate fertilizer & $F, R E E, C r, U$ & \\
\hline & & Iron-manganese & $\mathrm{Fe}, \mathrm{Mn}, \mathrm{Co}$ & Mn, Co & \\
\hline & & Superior iron & $\mathrm{Fe}$ & $M n$ & \\
\hline
\end{tabular}


[ \pm , present (absent); - , not applicable; ?, maybe; Ag, silver; Al, aluminum; As, arsenic; Au, gold; B, boron; Ba, barium; $\mathrm{Be}$, beryllium; $\mathrm{Bi}$, bismuth; $\mathrm{Br}$, bromine; $\mathrm{Ca}$, calcium; $\mathrm{Cd}$, cadmium; $\mathrm{Co}$, cobalt; $\mathrm{CO}$, carbon dioxide; Cs, cesium; Cr, chromium; Cu, copper; F, fluorine; Fe, iron; Ga, gallium; Ge, germanium; Hf, hafnium; Hg, mercury; I, iodine; IAEA, International Atomic Energy Agency; In, indium; IOA, iron

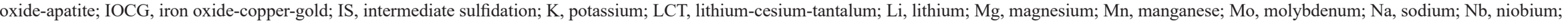

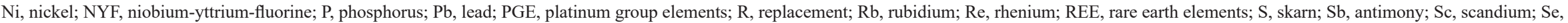

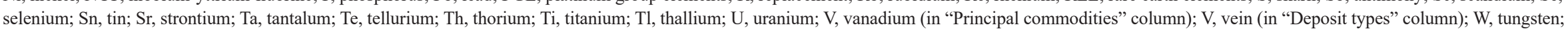
Y, yttrium; Zn, zinc; Zr, zirconium]

\begin{tabular}{|c|c|c|c|c|c|}
\hline System name & Synopsis & Deposit types & Principal commodities & Critical minerals 1 & Reference(s) \\
\hline \multirow[t]{2}{*}{ Petroleum } & \multirow{2}{*}{$\begin{array}{l}\mathrm{Ni} \text { and } \mathrm{V} \text { in poprhyrin complexes are } \\
\text { the most abundant metals in plant } \\
\text { and animal remains in source rocks } \\
\text { and in derived petroleum. Helium } \\
\text { is produced by radioactive decay } \\
\text { of U and Th in felsic igneous rocks } \\
\text { and siliciclastic rocks derived from } \\
\text { them. It is released by magmatic } \\
\text { heat and (or) fracturing and ac- } \\
\text { cumulates in gas reservoirs below an } \\
\text { impermeable seal. }\end{array}$} & Petroleum & Asphalt, Petroleum, V, Ni & V & \multirow[t]{2}{*}{$\begin{array}{l}\text { Magoon and Dow, } \\
\text { 1994; Hunt, 1996; } \\
\text { Brennan and East, } \\
\text { 2015; Craddock } \\
\text { and others, 2017; } \\
\text { King, 2020 }\end{array}$} \\
\hline & & Natural Gas & Natural gas, He & Helium & \\
\hline $\begin{array}{l}\text { Hybrid magmatic REE/ } \\
\text { basin brine path }\end{array}$ & $\begin{array}{l}\text { This hybrid system operates where } \\
\mathrm{CO}_{2} \text { - and HF-bearing magmatic vol- } \\
\text { atiles condense into basinal brines } \\
\text { that replace carbonate with fluorspar } \\
\pm \text { barite, } \mathrm{REE}, \mathrm{Ti}, \mathrm{Nb} \text {, and Be as } \\
\text { in the Illinois-Kentucky Fluorspar } \\
\text { District and Hicks Dome. }\end{array}$ & Fluorspar & Fluorite & $\begin{array}{l}\text { Fluorite, barite, } \\
\quad R E E, T i, N b, B e\end{array}$ & $\begin{array}{l}\text { Plumlee and others, } \\
\text { 1995; Denny and } \\
\text { others, 2015, } \\
\text { 2016; Hayes and } \\
\text { others, } 2017\end{array}$ \\
\hline Arsenide & $\begin{array}{l}\text { Arsenide systems form in continental } \\
\text { rifts where deep-seated, oxidized, } \\
\text { metal-rich, metamorphic basement } \\
\text { brines ascend to shallow levels. } \\
\text { Native elements (Ag, Bi, As), Ni-, } \\
\text { Co- and Fe-mono-, di- and sulf- } \\
\text { arsenides precipitate by reduction as } \\
\text { hydrocarbons, graphite, or sulfide } \\
\text { minerals are oxidized to form car- } \\
\text { bonates and barite. }\end{array}$ & Five element veins & $\mathrm{Ag}, \mathrm{As}, \mathrm{Co}, \mathrm{Ni}, \mathrm{Bi}, \mathrm{U}, \mathrm{Sb}$ & $\mathbf{C o}, \mathbf{B i}, \mathbf{U}, A s, S b$ & $\begin{array}{l}\text { Kissin, 1992, Markl } \\
\text { and others, 2016; } \\
\text { Burisch and others, } \\
\text { 2017; Scharrer and } \\
\text { others, } 2019\end{array}$ \\
\hline
\end{tabular}


Table 1. Systems-Deposits-Commodities-Critical Minerals Table for the Earth Mapping Resources Initiative.-Continued

[ \pm , present (absent); - , not applicable; ?, maybe; $\mathrm{Ag}$, silver; $\mathrm{Al}$, aluminum; $\mathrm{As}$, arsenic; $\mathrm{Au}$, gold; $\mathrm{B}$, boron; $\mathrm{Ba}$, barium; $\mathrm{Be}$, beryllium; $\mathrm{Bi}$, bismuth; $\mathrm{Br}$, bromine; $\mathrm{Ca}$, calcium; $\mathrm{Cd}$, cadmium; $\mathrm{Co}$, cobalt; $\mathrm{CO}$, carbon dioxide; Cs, cesium; Cr, chromium; Cu, copper; F, fluorine; Fe, iron; Ga, gallium; Ge, germanium; Hf, hafnium; Hg, mercury; I, iodine; IAEA, International Atomic Energy Agency; In, indium; IOA, iron

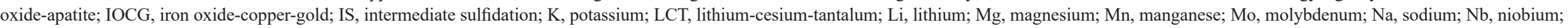

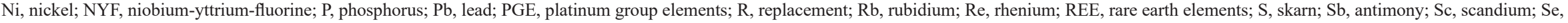
selenium; Sn, tin; Sr, strontium; Ta, tantalum; Te, tellurium; Th, thorium; Ti, titanium; Tl, thallium; U, uranium; V, vanadium (in "Principal commodities" column); V, vein (in "Deposit types" column); W, tungsten; Y, yttrium; Zn, zinc; Zr, zirconium]

\begin{tabular}{|c|c|c|c|c|c|}
\hline System name & Synopsis & Deposit types & Principal commodities & Critical minerals 1 & Reference(s) \\
\hline \multirow[t]{6}{*}{ Volcanogenic seafloor } & \multirow{6}{*}{$\begin{array}{l}\text { Volcanogenic seafloor systems are } \\
\text { driven by igneous activity along } \\
\text { spreading centers, back-arc basins } \\
\text { and magmatic arcs. In spread- } \\
\text { ing centers and back-arc basins, } \\
\text { seawater evolves to become an ore } \\
\text { fluid by convection through hot } \\
\text { volcanic rocks. In magmatic arcs, } \\
\text { ore fluids exsolved from subvolcanic } \\
\text { intrusions may mix with convecting } \\
\text { seawater. Ore deposits form where } \\
\text { hot reduced ore fluids vent into cool } \\
\text { oxygenated seawater. Sulfides and } \\
\text { sulfates precipitate in or near vents. } \\
\text { Mn and Fe precipitate at chemo- } \\
\text { clines over wide areas in basins with } \\
\text { seafloor hydrothermal activity. }\end{array}$} & Copper-zinc sulfide & $\mathrm{Cu}, \mathrm{Zn}$ & $\begin{array}{l}\text { Co, Bi, Te, In, Sn, } \\
\quad \text { Ge, Ga, Sb }\end{array}$ & \multirow{6}{*}{$\begin{array}{l}\text { Levson, 1995; } \\
\text { Shanks and } \\
\text { Thurston, 2012; } \\
\text { Monecke and } \\
\text { others, 2016; } \\
\text { Cannon and } \\
\text { others, 2017; DSM } \\
\text { Observer, 2020 }\end{array}$} \\
\hline & & Zinc-copper sulfide & $\mathrm{Zn}, \mathrm{Cu}$ & $\begin{array}{l}G e, G a, S b, C o, B i \\
\quad T e, I n, S n\end{array}$ & \\
\hline & & Polymetallic sulfide & $\mathrm{Cu}, \mathrm{Zn}, \mathrm{Pb}, \mathrm{Ag}, \mathrm{Au}$ & $\begin{array}{l}\text { Sn, Bi, Te, In, Ge, } \\
\quad \mathrm{Ga}, \mathrm{Sb}, \mathrm{As}\end{array}$ & \\
\hline & & Barite & Barite & Barite & \\
\hline & & $\begin{array}{l}\text { Manganese oxide (layers, crusts, } \\
\text { nodules) }\end{array}$ & $\mathrm{Mn}, \mathrm{Fe}, \mathrm{Ni}$ & $\begin{array}{l}\text { Mn, } C o, G e, T e, \\
\quad R E E, S c\end{array}$ & \\
\hline & & Algoma iron & $\mathrm{Fe}$ & $?$ & \\
\hline \multirow[t]{4}{*}{ Orogenic } & \multirow{4}{*}{$\begin{array}{l}\text { Metamorphic dewatering of sulfidic } \\
\text { volcanic and (or) sulfidic, carbona- } \\
\text { ceous, and (or) calcareous siliciclastic } \\
\text { sequences during exhumation with } \\
\text { fluid flow along dilatant structures. } \\
\text { Iron minerals in host rocks are often } \\
\text { sulfidized. Metavolcanic host rocks } \\
\text { often contain volcanogenic seafloor } \\
\text { sulfide deposits. }\end{array}$} & Gold & $\mathrm{Au}, \mathrm{Ag}$ & $\mathbf{W}, T e, A s, S b$ & \multirow{4}{*}{$\begin{array}{l}\text { Groves and others, } \\
\text { 1998; Gray and } \\
\text { Bailey, 2003; } \\
\text { Goldfarb and } \\
\text { others, 2005, } \\
\text { 2016; Luque and } \\
\text { others, } 2014\end{array}$} \\
\hline & & Antimony & $\mathrm{Sb}, \mathrm{Au}, \mathrm{Ag}$ & Sb & \\
\hline & & Mercury & $\mathrm{Hg}, \mathrm{Sb}$ & $S b$ & \\
\hline & & Graphite & Graphite (lump) & Graphite (lump) & \\
\hline \multirow[t]{3}{*}{ Coeur d'Alene-type } & \multirow{3}{*}{$\begin{array}{l}\text { Metamorphic dewatering of moderately } \\
\text { oxidized siliciclastic sequences dur- } \\
\text { ing exhumation with fluid flow along } \\
\text { dilatant structures. Metasedimentary } \\
\text { host rocks may contain basin brine } \\
\text { path } \mathrm{Pb}-\mathrm{Zn} \text { and } \mathrm{Cu} \pm \mathrm{Co} \text { deposits. }\end{array}$} & Polymetallic sulfide & $\mathrm{Ag}, \mathrm{Pb}, \mathrm{Zn}, \mathrm{Cu}$ & $\mathbf{S b}, \mathbf{C o}, G e, G a$, In & \multirow{3}{*}{$\begin{array}{l}\text { Wallace and Whelan, } \\
\text { 1986; Leach and } \\
\text { others, 1988, 1998; } \\
\text { Beaudoin and } \\
\text { Sangster, 1992, } \\
\text { 1996; Balistrieri } \\
\text { and others, 2002; } \\
\text { Zartman and Smith, } \\
\text { 2009; Hofstra and } \\
\text { others, 2013a; Seal } \\
\text { and others, 2017; } \\
\text { IAEA, 2020 }\end{array}$} \\
\hline & & Antimony & $\mathrm{Sb}$ & Sb & \\
\hline & & Uranium & $\mathrm{U}$ & $\mathbf{U}$ & \\
\hline
\end{tabular}




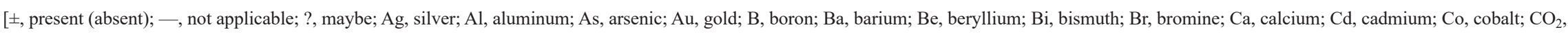
carbon dioxide; Cs, cesium; Cr, chromium; Cu, copper; F, fluorine; Fe, iron; Ga, gallium; Ge, germanium; Hf, hafnium; Hg, mercury; I, iodine; IAEA, International Atomic Energy Agency; In, indium; IOA, iron

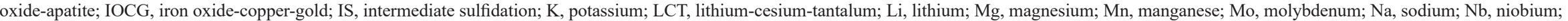

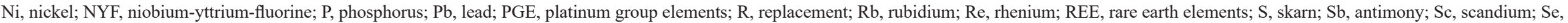

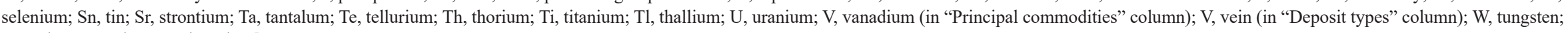
Y, yttrium; Zn, zinc; Zr, zirconium]

\begin{tabular}{|c|c|c|c|c|c|}
\hline System name & Synopsis & Deposit types & Principal commodities & Critical minerals' 1 & Reference(s) \\
\hline \multirow[t]{4}{*}{ Metamorphic } & \multirow[t]{4}{*}{$\begin{array}{l}\text { Metamorphic systems recrystallize } \\
\text { rocks containing organic carbon or } \\
\text { REE phosphate minerals or uranium } \\
\text { minerals. Crystalline magnesite } \\
\text { forms by carbonation of peridotite. }\end{array}$} & Graphite (coal or carbonaceous sed) & Graphite (amorphous and flake) & $\begin{array}{l}\text { Graphite (amor- } \\
\text { phous and } \\
\text { flake) }\end{array}$ & \multirow{4}{*}{$\begin{array}{l}\text { Sutphin, 1991a,b,c; } \\
\text { Luque and others, } \\
\text { 2014; McKinney } \\
\text { and others, 2015; } \\
\text { Sutherland and } \\
\text { Cola, 2016; Rob- } \\
\text { inson and others, } \\
\text { 2017; Menzel } \\
\text { and others, } 2018 \text {; } \\
\text { IAEA, } 2020\end{array}$} \\
\hline & & Magnesite & $\mathrm{Mg}$ & Mg & \\
\hline & & Gneiss REE (monazite, xenotime) & Th, U, REE, Y & REE, $U$ & \\
\hline & & Gneiss uranium & $\mathrm{U}$ & $\mathbf{U}$ & \\
\hline \multirow[t]{13}{*}{ Porphyry $\mathrm{Cu}-\mathrm{Mo}-\mathrm{Au}$} & \multirow{13}{*}{$\begin{array}{l}\text { Porphyry copper-molybdenum-gold } \\
\text { systems operate in oceanic and } \\
\text { continental magmatic arcs with } \\
\text { calc-alkaline compositions. Aqueous } \\
\text { supercritical fluids exsolved from } \\
\text { felsic plutons and the apices of } \\
\text { subvolcanic stocks form a variety of } \\
\text { deposit types as they move upward } \\
\text { and outward, split into liquid and } \\
\text { vapor, react with country rocks, and } \\
\text { mix with groundwater. The broad } \\
\text { spectrum of deposit types results } \\
\text { from the large thermal and chemical } \\
\text { gradients in these systems. }\end{array}$} & Greisen & Mo, W, Sn & $W, S n$ & \multirow{13}{*}{$\begin{array}{l}\text { Seedorff and others, } \\
\text { 2005; John and } \\
\text { others, 2010, } \\
\text { 2017; Sillitoe, } \\
\text { 2010; Taylor and } \\
\text { others, 2012; John } \\
\text { and Taylor, 2016; } \\
\text { London, 2016; } \\
\text { Wang and others, } \\
2021\end{array}$} \\
\hline & & S-R-V tungsten & $\mathrm{W}$ & $\mathbf{W}, \mathbf{B i}, M n, S c$ & \\
\hline & & Porphyry/skarn molybdenum & Mo, W, Sn & $W, R e, B i$ & \\
\hline & & Porphyry/skarn copper & $\mathrm{Cu}, \mathrm{Au}, \mathrm{Ag}, \mathrm{Mo}$ & $\begin{array}{l}\text { PGE, Te, Re, U, } \\
\text { Sc, } C o, B i\end{array}$ & \\
\hline & & Skarn iron & $\mathrm{Fe}, \mathrm{Cu}$ & $G e$ & \\
\hline & & Skarn magnesite & $\mathrm{Mg}$ & Mg & \\
\hline & & $\mathrm{R}-\mathrm{V}$ manganese & $\mathrm{Mn}$ & Mn, Co & \\
\hline & & Polymetallic sulfide S-R-V-IS & $\mathrm{Cu}, \mathrm{Zn}, \mathrm{Cd}, \mathrm{Pb}, \mathrm{Ag}, \mathrm{Au}$ & $\begin{array}{c}M n, G e, G a, I n, B i \\
\quad S b, A s, W, T e\end{array}$ & \\
\hline & & Distal disseminated silver-gold & $\mathrm{Ag}, \mathrm{Au}$ & $S b, A s$ & \\
\hline & & High-sulfidation gold-silver & $\mathrm{Cu}, \mathrm{Ag}, \mathrm{Au}$ & $\begin{array}{l}A s, S b, T e, B i, S n \\
\quad G a\end{array}$ & \\
\hline & & Intermediate sulfidation antimony & $\mathrm{Sb}, \mathrm{Hg}$ & Sb, $A s$ & \\
\hline & & Lithocap alunite & $\mathrm{Al}, \mathrm{K}_{2} \mathrm{SO}_{4}$ (potash) & $\mathrm{Al}, \mathrm{K}_{2} \mathrm{SO}_{4}, \mathrm{Ga}$ & \\
\hline & & Lithocap kaolinite & Kaolin & $G a$ & \\
\hline
\end{tabular}


Table 1. Systems-Deposits-Commodities-Critical Minerals Table for the Earth Mapping Resources Initiative.-Continued

[ \pm , present (absent); - , not applicable; ?, maybe; $\mathrm{Ag}$, silver; $\mathrm{Al}$, aluminum; As, arsenic; $\mathrm{Au}$, gold; $\mathrm{B}$, boron; $\mathrm{Ba}$, barium; $\mathrm{Be}$, beryllium; $\mathrm{Bi}$, bismuth; $\mathrm{Br}$, bromine; $\mathrm{Ca}$, calcium; $\mathrm{Cd}$, cadmium; $\mathrm{Co}$, cobalt; $\mathrm{CO}$, carbon dioxide; Cs, cesium; Cr, chromium; Cu, copper; F, fluorine; Fe, iron; Ga, gallium; Ge, germanium; Hf, hafnium; Hg, mercury; I, iodine; IAEA, International Atomic Energy Agency; In, indium; IOA, iron oxide-apatite; IOCG, iron oxide-copper-gold; IS, intermediate sulfidation; K, potassium; LCT, lithium-cesium-tantalum; Li, lithium; Mg, magnesium; Mn, manganese; Mo, molybdenum; Na, sodium; Nb, niobium;

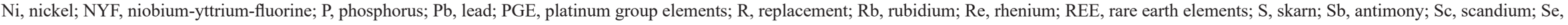
selenium; Sn, tin; Sr, strontium; Ta, tantalum; Te, tellurium; Th, thorium; Ti, titanium; Tl, thallium; U, uranium; V, vanadium (in "Principal commodities" column); V, vein (in "Deposit types" column); W, tungsten; Y, yttrium; Zn, zinc; Zr, zirconium]

\begin{tabular}{|c|c|c|c|c|c|}
\hline System name & Synopsis & Deposit types & Principal commodities & Critical minerals ${ }^{1}$ & Reference(s) \\
\hline \multirow[t]{10}{*}{ Alkalic porphyry } & \multirow{10}{*}{$\begin{array}{l}\text { Alkalic porphyry systems form in oce- } \\
\text { anic and continental magmatic arcs } \\
\text { and in continental rifts by similar } \\
\text { processes from fluids exsolved from } \\
\text { more fractionated alkalic plutons } \\
\text { and stocks. Resulting ore deposits } \\
\text { tend to be more enriched in } \mathrm{Au}, \mathrm{Te}, \\
\mathrm{Bi} \text {, and } \mathrm{V} \text {. }\end{array}$} & Greisen & Mo, Bi & $\mathrm{Bi}$ & \multirow{10}{*}{$\begin{array}{l}\text { Jensen and Barton, } \\
\text { 2000; Kelley and } \\
\text { Spry, 2016; Wang } \\
\text { and others, } 2021\end{array}$} \\
\hline & & S-R-V Tungsten & $\mathrm{W}$ & $\mathbf{W}, B i, M n, S c$ & \\
\hline & & Porphyry/skarn copper-gold & $\mathrm{Cu}, \mathrm{Mo}, \mathrm{Au}$ & $P G E, T e, B i$ & \\
\hline & & Polymetallic sulfide S-R-V-IS & $\mathrm{Au}, \mathrm{Ag}, \mathrm{Pb}, \mathrm{Zn}, \mathrm{Cu}$ & $G e, G a, I n, B i, T e$ & \\
\hline & & Fluorspar & Fluorite & Fluorite & \\
\hline & & Distal disseminated silver-gold & $\mathrm{Ag}, \mathrm{Au}$ & $S b, A s$ & \\
\hline & & High sulfidation & $\mathrm{Cu}, \mathrm{Ag}, \mathrm{Au}$ & $T e, B i, A s, S b$ & \\
\hline & & Low sulfidation & $\mathrm{Au}$ & $T e, B i, V, F$ & \\
\hline & & Lithocap alunite? & $\mathrm{Al}, \mathrm{K}_{2} \mathrm{SO}_{4}$ (potash) & $\mathrm{Al}, \mathrm{K}_{2} \mathrm{SO}_{4}, \mathrm{Ga}$ & \\
\hline & & Lithocap kaolinite? & Kaolin & $G a$ & \\
\hline \multirow[t]{8}{*}{$\begin{array}{l}\text { Porphyry Sn (granite- } \\
\text { related) }\end{array}$} & \multirow{8}{*}{$\begin{array}{l}\text { Granite-related porphyry Sn systems } \\
\text { form in back-arc or hinterland } \\
\text { settings by similar processes from } \\
\text { fluids exsolved from more crustally } \\
\text { contaminated S-type peraluminous } \\
\text { plutons and stocks. At deep levels, } \\
\text { LCT pegmatites emanate from } \\
\text { plutons. Resulting ore deposits tend } \\
\text { to be } \mathrm{Cu} \text { and } \mathrm{Mo} \text { poor and enriched } \\
\text { in } \mathrm{Li}, \mathrm{Cs}, \mathrm{Ta}, \mathrm{Nb}, \mathrm{Sn}, \mathrm{W}, \mathrm{Ag}, \mathrm{Sb}, \\
\text { and } \mathrm{In} .\end{array}$} & Pegmatite LCT & Li-Cs-Ta & $\begin{array}{l}\text { Li, Cs, Ta, Nb, Sn, } \\
\text { Be, } S c\end{array}$ & \multirow{8}{*}{$\begin{array}{l}\text { Panteleyev, 1996; } \\
\text { Sillitoe and others, } \\
\text { 1998; Černý and } \\
\text { Ercit, 2005; Martin } \\
\text { and De Vito, 2005; } \\
\text { London, 2008, } \\
\text { 2016; Bradley } \\
\text { and others, 2017a; } \\
\text { Kamilli and others, } \\
\text { 2017; Hulsbosch, } \\
\text { 2019; Wang and } \\
\text { others, } 2021\end{array}$} \\
\hline & & Greisen & $\mathrm{Sn}, \mathrm{W}, \mathrm{Be}$ & Sn, W, Be, $S c$ & \\
\hline & & Porphyry/skarn & Sn, W, Be & Sn, W, Be, $S c$ & \\
\hline & & Polymetallic sulfide S-R-V-IS & $\mathrm{Cu}, \mathrm{Zn}, \mathrm{Pb}, \mathrm{Ag}, \mathrm{Au}$ & $\begin{array}{l}\text { Sn, Mn, Ge, Ga, In, } \\
\quad B i, S b, A s\end{array}$ & \\
\hline & & Distal disseminated silver-gold & $\mathrm{Ag}, \mathrm{Au}$ & $S b, A s$ & \\
\hline & & High sulfidation & $\mathrm{Cu}, \mathrm{Ag}, \mathrm{Au}$ & $S n, S b, A s, T e, B i$ & \\
\hline & & Lithocap alunite & $\mathrm{Al}, \mathrm{K}_{2} \mathrm{SO}_{4}$ (potash) & $\mathrm{Al}, \mathrm{K}_{2} \mathrm{SO}_{4}, \mathrm{Ga}$ & \\
\hline & & Lithocap kaolinite & Kaolin & $G a$ & \\
\hline
\end{tabular}


[ \pm , present (absent); - , not applicable; ?, maybe; Ag, silver; Al, aluminum; As, arsenic; Au, gold; B, boron; Ba, barium; $\mathrm{Be}$, beryllium; $\mathrm{Bi}$, bismuth; $\mathrm{Br}$, bromine; $\mathrm{Ca}$, calcium; $\mathrm{Cd}$, cadmium; $\mathrm{Co}$, cobalt; $\mathrm{CO}$, carbon dioxide; Cs, cesium; Cr, chromium; Cu, copper; F, fluorine; Fe, iron; Ga, gallium; Ge, germanium; Hf, hafnium; Hg, mercury; I, iodine; IAEA, International Atomic Energy Agency; In, indium; IOA, iron

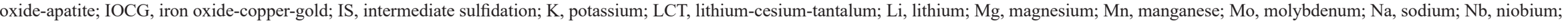

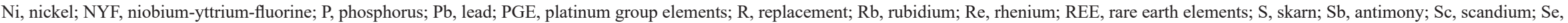

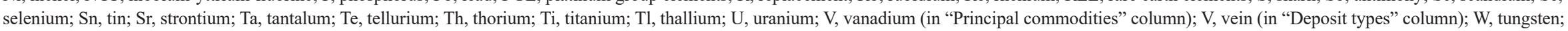
Y, yttrium; Zn, zinc; Zr, zirconium]

\begin{tabular}{|c|c|c|c|c|c|}
\hline System name & Synopsis & Deposit types & Principal commodities & Critical minerals 1 & Reference(s) \\
\hline \multirow[t]{9}{*}{ Reduced intrusion-related } & \multirow{9}{*}{$\begin{array}{l}\text { Reduced intrusion-related systems } \\
\text { form in continental magmatic arcs } \\
\text { by similar processes from fluids } \\
\text { exsolved from calc-alkaline plutons } \\
\text { and stocks that assimilated car- } \\
\text { bonaceous pyritic country rocks. } \\
\text { Resulting ore deposits tend to be } \\
\text { poor in } \mathrm{Cu}, \mathrm{Mo} \text {, and } \mathrm{Sn} \text { and enriched } \\
\text { in } \mathrm{W}, \mathrm{Au}, \mathrm{Ag}, \mathrm{Te}, \mathrm{Bi}, \mathrm{Sb} \text {, and As. }\end{array}$} & Pegmatite LCT & Li-Cs-Ta & $\begin{array}{l}\text { Li, Cs, Ta, Nb, Sn, } \\
\quad \text { Be, } S c\end{array}$ & \multirow{9}{*}{$\begin{array}{l}\text { Hart, 2007; Nutt and } \\
\text { Hofstra, 2007; } \\
\text { Luque and others, } \\
\text { 2014; Wang and } \\
\text { others, } 2021\end{array}$} \\
\hline & & Gold & $\mathrm{Au}, \mathrm{Ag}$ & $T e, B i, S b, A s$ & \\
\hline & & Greisen-V tungsten & W, Mo & $\mathbf{W}, S c$ & \\
\hline & & $\begin{array}{l}\begin{array}{l}\text { Skarn copper-molybdenum- } \\
\text { tungsten }\end{array} \\
\end{array}$ & $\mathrm{W}, \mathrm{Mo}, \mathrm{Cu}, \mathrm{Au}, \mathrm{Ag}$ & $W, T e, B i, R e$ & \\
\hline & & Polymetallic sulfide S-R-V-IS & $\mathrm{Au}, \mathrm{Ag}, \mathrm{Pb}, \mathrm{Zn}, \mathrm{Cu}$ & $\begin{array}{l}\text { Mn, Ge, Ga, In, Bi, } \\
\quad \text { Sb, As }\end{array}$ & \\
\hline & & Distal disseminated silver-gold & $\mathrm{Ag}, \mathrm{Au}$ & $T e, B i, S b, A s$ & \\
\hline & & Intermediate sulfidation & $\mathrm{Au}, \mathrm{Ag}, \mathrm{Pb}, \mathrm{Zn}, \mathrm{Cu}$ & $\begin{array}{l}\text { Mn, Ge, Ga, In, Bi, } \\
\quad \mathrm{Sb}, \mathrm{As}\end{array}$ & \\
\hline & & Intermediate sulfidation antimony & $\mathrm{Sb}$ & $S b$ & \\
\hline & & Graphite & Graphite (lump) & Graphite (lump) & \\
\hline \multirow[t]{3}{*}{ Carlin-type } & \multirow[b]{3}{*}{\begin{tabular}{|l} 
Carlin-type systems occur in continen- \\
tal magmatic arcs but are remote \\
from subjacent stocks and plutons. \\
Consequently, ore fluids consist \\
largely of meteoric water contain- \\
ing volatiles discharged from deep \\
intrusions. Ore fluids scavenge \\
elements from carbonaceous pyritic \\
sedimentary rocks as they convect \\
through them. Gold ore containing \\
disseminated pyrite forms where \\
acidic reduced fluids dissolve carbon- \\
ate and sulfidize Fe-bearing minerals \\
in host rocks. As, Hg, and Tl minerals \\
precipitate by cooling. Stibnite pre- \\
cipitates with quartz by cooling from \\
Au-, As-, Hg-, and Tl-depleted fluids.
\end{tabular}} & Gold & $\mathrm{Au}, \mathrm{Ag}, \mathrm{Hg}$ & $A s, S b$ & \multirow{3}{*}{$\begin{array}{l}\text { Hofstra and Cline, } \\
\text { 2000; Goldfarb } \\
\text { and others, 2016; } \\
\text { Muntean, } 2018\end{array}$} \\
\hline & & Antimony & $\mathrm{Sb}$ & Sb & \\
\hline & & Arsenic-thallium-mercury & As, $\mathrm{Tl}, \mathrm{Hg}$ & As & \\
\hline
\end{tabular}


Table 1. Systems-Deposits-Commodities-Critical Minerals Table for the Earth Mapping Resources Initiative.-Continued

[ \pm , present (absent); - , not applicable; ?, maybe; $\mathrm{Ag}$, silver; $\mathrm{Al}$, aluminum; $\mathrm{As}$, arsenic; $\mathrm{Au}$, gold; $\mathrm{B}$, boron; $\mathrm{Ba}$, barium; $\mathrm{Be}$, beryllium; $\mathrm{Bi}$, bismuth; $\mathrm{Br}$, bromine; $\mathrm{Ca}$, calcium; $\mathrm{Cd}$, cadmium; $\mathrm{Co}$, cobalt; $\mathrm{CO}$, carbon dioxide; Cs, cesium; Cr, chromium; Cu, copper; F, fluorine; Fe, iron; Ga, gallium; Ge, germanium; Hf, hafnium; Hg, mercury; I, iodine; IAEA, International Atomic Energy Agency; In, indium; IOA, iron

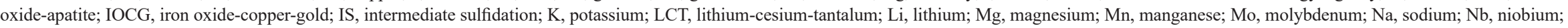

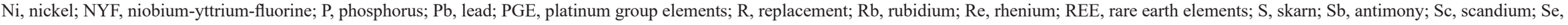
selenium; Sn, tin; Sr, strontium; Ta, tantalum; Te, tellurium; Th, thorium; Ti, titanium; Tl, thallium; U, uranium; V, vanadium (in "Principal commodities" column); V, vein (in "Deposit types" column); W, tungsten; Y, yttrium; $\mathrm{Zn}$, zinc; $\mathrm{Zr}$, zirconium]

\begin{tabular}{|c|c|c|c|c|c|}
\hline System name & Synopsis & Deposit types & Principal commodities & Critical minerals 1 & Reference(s) \\
\hline \multirow[t]{14}{*}{ Climax-type } & \multirow{14}{*}{$\begin{array}{l}\text { Climax-type systems occur in conti- } \\
\text { nental rifts with hydrous bimodal } \\
\text { magmatism. Aqueous supercritical } \\
\text { fluids exsolved from A-type topaz } \\
\text { rhyolite plutons, and the apices of } \\
\text { subvolcanic stocks form a variety of } \\
\text { deposit types as they move upward } \\
\text { and outward, split into liquid and } \\
\text { vapor, react with country rocks, and } \\
\text { mix with groundwater. The broad } \\
\text { spectrum of deposit types results } \\
\text { from the large thermal and chemical } \\
\text { gradients in these systems. At deep } \\
\text { levels, NYF pegmatites emanate } \\
\text { from plutons. }\end{array}$} & Pegmatite NYF & $\mathrm{Nb}, \mathrm{Y}, \mathrm{F}, \mathrm{Be}$ & $\mathrm{Nb}, \mathrm{Ta}, \mathrm{Be}, \mathrm{Sc}$ & \multirow{14}{*}{$\begin{array}{l}\text { Černý and Ercit, } \\
\text { 2005; Martin and } \\
\text { De Vito, 2005; } \\
\text { London, 2008, } \\
\text { 2016; Ludington } \\
\text { and Plumlee, } \\
\text { 2009; Breit and } \\
\text { Hall, 2011; Foley } \\
\text { and others, 2012; } \\
\text { Hofstra and others, } \\
\text { 2014; London, } \\
\text { 2016; Audétat and } \\
\text { Li, 2017; Wang } \\
\text { and others, 2021 }\end{array}$} \\
\hline & & Greisen & Mo, W, Sn & $\mathbf{W}, \boldsymbol{S n}, B i, B e, S c$ & \\
\hline & & Porphyry molybdenum & Mo, W, Sn & W, Sn, Re, REE, $S c$ & \\
\hline & & Skarn molybdenum & Mo, W, Sn & $\mathbf{W}, \mathbf{S n}, B e, S c$ & \\
\hline & & Greisen-S-R berylium & $\mathrm{Be}, \mathrm{F}$ & $\mathrm{Be}, \mathrm{F}$ & \\
\hline & & Polymetallic sulfide S-R-V-IS & $\mathrm{Cu}, \mathrm{Zn}, \mathrm{Pb}, \mathrm{Ag}, \mathrm{Au}$ & $\begin{array}{l}\text { Mn, Ge, Ga, In, Bi, } \\
\quad \text { Sb, As }\end{array}$ & \\
\hline & & Distal disseminated silver-gold & $\mathrm{Ag}, \mathrm{Au}$ & $S b, A s$ & \\
\hline & & High sulfidation & $\mathrm{Cu}, \mathrm{Ag}, \mathrm{Au}$ & $S n, S b, A s, T e, B i$ & \\
\hline & & Lithocap alunite & $\mathrm{Al}, \mathrm{K}_{2} \mathrm{SO}_{4}$ (potash) & $\mathrm{Al}, \mathrm{K}_{2} \mathrm{SO}_{4}, G a$ & \\
\hline & & Lithocap kaolinite & Kaolin & $G a$ & \\
\hline & & Fluorspar & Fluorite & Fluorite & \\
\hline & & Volcanogenic beryllium & $\mathrm{Be}, \mathrm{U}$ & Be, $U, L i$ & \\
\hline & & Volcanogenic uranium & $\mathrm{U}$ & $\mathbf{U}, L i, B e$ & \\
\hline & & Rhoylite tin & $\mathrm{Sn}$ & Sn & \\
\hline \multirow[t]{7}{*}{ IOA-IOCG } & \multirow{7}{*}{$\begin{array}{l}\text { IOA-IOCG systems form in both } \\
\text { subduction- and rift-related mag- } \\
\text { matic provinces. IOA deposits } \\
\text { form as hot brine discharged from } \\
\text { subvolcanic mafic to intermedi- } \\
\text { ate composition intrusions reacts } \\
\text { with cool country rocks. Albitite } \\
\text { uranium deposits form at deeper } \\
\text { levels where brines albitize country } \\
\text { rocks. IOCG deposits form on the } \\
\text { roof or periphery of IOA mineraliza- } \\
\text { tion at lower temperatures, often } \\
\text { with involvement of external fluids. } \\
\text { Polymetallic skarn, replacement, and } \\
\text { vein deposits occur outboard from } \\
\text { IOCG deposits. Mn replacement and } \\
\text { lacustrine Fe deposits form near or } \\
\text { at the paleosurface. }\end{array}$} & Albitite uranium & $\mathrm{U}$ & $\mathbf{U}$ & \multirow{7}{*}{$\begin{array}{l}\text { Williams and others, } \\
\text { 2005; Cox and } \\
\text { Singer, 2007; } \\
\text { Groves and others, } \\
\text { 2010; Slack, 2013; } \\
\text { Barton, 2014; } \\
\text { Slack and others, } \\
2016\end{array}$} \\
\hline & & Iron oxide apatite & $\mathrm{Fe}$ & $R E E$ & \\
\hline & & Iron oxide copper gold & $\mathrm{Cu}, \mathrm{Au}, \mathrm{U}, \mathrm{Co}, \mathrm{Se}$ & $\mathbf{U}, C o$ & \\
\hline & & Skarn iron & $\mathrm{Fe}, \mathrm{P}$ & REE, Ge & \\
\hline & & Polymetallic sulfide S-R-V & $\mathrm{Ni}, \mathrm{Co}, \mathrm{Mo}, \mathrm{Cu}, \mathrm{Zn}, \mathrm{Pb}, \mathrm{Ag}, \mathrm{Au}$ & $\begin{array}{l}\text { Co, } R e, G e, G a, I n \\
\quad B i, T e, S b, A s\end{array}$ & \\
\hline & & Replacement manganese & $\mathrm{Mn}$ & Mn, Co & \\
\hline & & Lacustrine iron & $\mathrm{Fe}$ & - & \\
\hline
\end{tabular}


[ \pm , present (absent); - , not applicable; ?, maybe; Ag, silver; Al, aluminum; As, arsenic; Au, gold; B, boron; Ba, barium; $\mathrm{Be}$, beryllium; $\mathrm{Bi}$, bismuth; $\mathrm{Br}$, bromine; $\mathrm{Ca}$, calcium; $\mathrm{Cd}$, cadmium; $\mathrm{Co}$, cobalt; $\mathrm{CO}$, carbon dioxide; Cs, cesium; Cr, chromium; Cu, copper; F, fluorine; Fe, iron; Ga, gallium; Ge, germanium; Hf, hafnium; Hg, mercury; I, iodine; IAEA, International Atomic Energy Agency; In, indium; IOA, iron

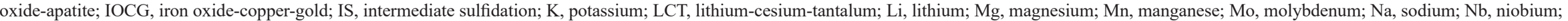

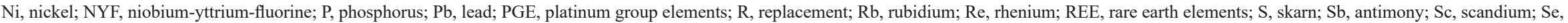

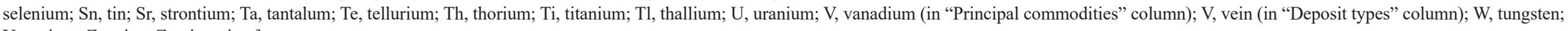
Y, yttrium; Zn, zinc; Zr, zirconium]

\begin{tabular}{|c|c|c|c|c|c|}
\hline System name & Synopsis & Deposit types & Principal commodities & Critical minerals 1 & Reference(s) \\
\hline \multirow[t]{4}{*}{ Magmatic REE } & \multirow{4}{*}{$\begin{array}{l}\text { Magmatic REE systems typically } \\
\text { occur in continental rifts or along } \\
\text { translithospheric structures. REE and } \\
\text { other elements in mantle-derived } \\
\text { ultrabasic, alkaline, and peralkaline } \\
\text { (agpaitic) intrusions are enriched } \\
\text { by fractionation and separation of } \\
\text { immiscible carbonatite melts } \pm \text { saline } \\
\text { hydrothermal liquids. Exsolved mag- } \\
\text { matic fluids or heated external fluids } \\
\text { may deposit REE and other elements } \\
\text { in adjacent country rocks. }\end{array}$} & $\begin{array}{l}\text { Peralkaline syenite/granite/rhyo- } \\
\text { lite/ alaskite/pegmatites }\end{array}$ & $\begin{array}{l}\text { REE, Y, Zr, Hf, Nb, Ta, Be, U, } \\
\quad \text { Th, } \mathrm{Cu}\end{array}$ & $\begin{array}{l}\text { REE, Zr, Hf, Nb, } \\
\text { Ta, Be, U, V, Te, } \\
\text { fluorite }\end{array}$ & \multirow{4}{*}{$\begin{array}{l}\text { Verplanck and others, } \\
\text { 2014, 2016; } \\
\text { Dostal, 2016, } \\
\text { 2017; Wang and } \\
\text { others, } 2021\end{array}$} \\
\hline & & Carbonatite & $\begin{array}{l}\text { REE, P, Y, Nb, Ba, Sr, U, Th, } \\
\quad \mathrm{Cu}\end{array}$ & $\begin{array}{l}\text { REE, Nb, Sc, U, } \\
\text { Sr, Ba, P, Cu, } \\
\text { Zr, magnetite, } \\
\text { vermiculite, } \\
\text { fluorite } \\
\end{array}$ & \\
\hline & & Phosphate & REE, $P$ & $R E E$ & \\
\hline & & Fluorspar & Flourite & $\begin{array}{l}\text { Flourite, barite, } T i \text {, } \\
\quad N b, Z r, R E E, S c \text {, } \\
\quad U, B e\end{array}$ & \\
\hline \multirow[t]{4}{*}{ Mafic magmatic } & \multirow{4}{*}{$\begin{array}{l}\text { Mafic magmatic systems generally } \\
\text { form in large igneous provinces re- } \\
\text { lated to mantle plumes or meteorite } \\
\text { impacts. Nickel-copper sulfide ores } \\
\text { with PGEs result from settling and } \\
\text { accumulation of immiscible sulfide } \\
\text { liquids in mafic layered intrusions } \\
\text { and ultramafic magma conduits. In } \\
\text { layered intrusions, Fe-Ti oxides, } \\
\text { chromite, and PGE minerals crystal- } \\
\text { ize from evolving parental magmas } \\
\text { and are concentrated by physical } \\
\text { processes in cumulate layers. In } \\
\text { anorthosites, Fe-Ti oxides } \pm \text { apatite } \\
\text { crystalize from residual magmas } \\
\text { entrained in plagioclase-melt diapirs. } \\
\text { In convergent settings, Alaskan-type } \\
\text { intrusions with Fe-Ti oxides and } \\
\text { PGE form from mantle melts. }\end{array}$} & Chromite & $\mathrm{Cr}$ & $\mathbf{C r}$ & \multirow{4}{*}{$\begin{array}{l}\text { Ash, 1996; Schulte } \\
\text { and others, 2012; } \\
\text { Ernst and Jowitt, } \\
\text { 2013; Woodruff } \\
\text { and others, 2013; } \\
\text { Zientek and others, } \\
\text { 2017; Mondal and } \\
\text { Griffin, } 2018\end{array}$} \\
\hline & & Nickel-copper-PGE sulfide & $\mathrm{Ni}, \mathrm{Cu}, \mathrm{Co}, \mathrm{PGE}, \mathrm{Ag}, \mathrm{Au}, \mathrm{Se}, \mathrm{Te}$ & Co, PGE, $T e$ & \\
\hline & & PGE (low sulfide) & PGE & PGE & \\
\hline & & Iron-titanium oxide & Fe, Ti, V, P & $\mathbf{T i}, \mathbf{V}, R E E$ & \\
\hline
\end{tabular}

${ }^{1}$ Elements in bold have been produced from some deposits, whereas those in italics are potential critical minerals. 


\section{References Cited}

Alpine, A.E., ed., 2010, Hydrological, geological, and biological site characterization of breccia pipe uranium deposits in northern Arizona: U.S. Geological Survey Scientific Investigations Report 2010-5025, 353 p., 1 pl., scale 1:375,000, accessed April 18, 2020, at https://doi.org/ 10.3133/sir20105025.

Ash, C., 1996, Podiform chromite, in Lefebure, D.V., and Hõy, T., eds., Selected British Columbia mineral deposit profiles, volume 2-Metallic deposits: British Columbia Ministry of Employment and Investment, Open File 1996-13, p. 109-112.

Audétat, A., and Li, W., 2017, The genesis of Climaxtype porphyry Mo deposits - Insights from fluid inclusions and melt inclusions: Ore Geology Reviews, v. 88, p. 436-460. [Also available at https://doi.org/10.1016/ j.oregeorev.2017.05.018.]

Balistrieri, L.S., Box, S.E., and Bookstrom, A.A., 2002, A geoenvironmental model for polymetallic vein deposits-A case study in the Coeur d'Alene mining district and comparisons with drainage from mineralized deposits in the Colorado Mineral Belt and Humboldt Basin, Nevada, in Seal, R.R., II, and Foley, N.K., eds., Progress on geoenvironmental models of mineral deposits: U.S. Geological Survey Open-File Report 02-195, p. 143-160.

Barton, M.D., 2014, Iron oxide(-Cu-Au-REE-P-Ag-U-Co) systems, chap. 13.20 of Heinrich, D.H., and Turekian, K.K., eds., Treatise on geochemistry, second edition: Amsterdam, Elsevier Ltd., p. 515-541, accessed April 18, 2020, at https://doi.org/10.1016/B978-0-08-095975-7.01123-2.

Beaudoin, G., and Sangster, D.F., 1992, A descriptive model for silver-lead-zinc veins in clastic metasedimentary terranes: Economic Geology, v. 87, no. 4, p. 1005-1021, accessed April 18, 2020, at https://doi.org/10.2113/ gsecongeo.87.4.1005.

Beaudoin, G., and Sangster, D.F., 1995, Clastic metasedimenthosted vein silver-lead-zinc, in Eckstrand, O.R., Sinclair, W.D., and Thorpe, R.I., eds., Geology of Canadian mineral deposit types: Geological Survey of Canada, Geology of Canada 8, p. 393-398. [Also available at https://doi.org/ 10.1130/DNAG-GNA-P1.393.]

Bradley, D.C., McCauley, A.D., and Stillings, L.M., 2017a, Mineral-deposit model for lithium-cesium-tantalum pegmatites: U.S. Geological Survey Scientific Investigations Report 2010-5070-O, 48 p., accessed April 18, 2020, at https://doi.org/10.3133/sir201050700.
Bradley, D.C., Munk, L., Jochens, H., Hynek, S., and Labay, K., 2013, A preliminary deposit model for lithium brines: U.S. Geological Survey Open-File Report 2013-1006, 6 p., accessed April 18, 2020, at https://doi.org/10.3133/ ofr20131006.

Bradley, D.C., Stillings, L.L., Jaskula, B.W., Munk, L., and McCauley, A.D., 2017b, Lithium, chap. K of Schulz, K.J., DeYoung, J.H., Jr., Seal, R.R., II, and Bradley, D.C., eds., Critical mineral resources of the United States-Economic and environmental geology and prospects for future supply: U.S. Geological Survey Professional Paper 1802, p. K1-K21, accessed April 18, 2020, at https://doi.org/10.3133/pp1802K.

Breit, G.N., 2016, Resource potential for commodities in addition to uranium in sandstone-hosted deposits, chap. 13 of Verplanck, P.L., and Hitzman, M.W., eds., Reviews in economic geology, volume 18-Rare earth and critical elements in ore deposits: Littleton, Colo., Society of Economic Geologists, Inc., p. 323-338. [Also available at https://doi.org/10.5382/Rev.18.13.]

Breit, G.N., and Hall, S.M., 2011, Deposit model for volcanogenic uranium deposits: U.S. Geological Survey OpenFile Report 2011-1255, 5 p., accessed April 18, 2020, at https://doi.org/10.3133/ofr20111255.

Brennan, S.T., and East, J.A., II, 2015, The U.S. Geological Survey national helium resource assessment [abs.], in American Geophysical Union Fall Meeting, 2015, San Francisco, Calif., December 14-18, 2015, Abstracts: American Geophysical Union, abstract no. IN33a-1789, 1 p., accessed March 30, 2021, at https://ui.adsabs.harvard.edu/ abs/2015AGUFMIN33A1789B/abstract.

Bruneton, P., and Cuney, M., 2016, Geology of uranium deposits, chap. 2 of Hore-Lacy, I., ed., Uranium for nuclear power-Resources, mining, and transformation to fuel: Cambridge, Mass., Woodhead Publishing, p. 11-52.

Burisch, M., Gerdes, A., Walter, B.F., Neumann, U., Fettel, M., and Markl, G., 2017, Methane and the origin of fiveelement veins-Mineralogy, age, fluid inclusion chemistry and ore forming processes in the Odenwald, SW Germany: Ore Geology Reviews, v. 81, p. 42-61, accessed April 18, 2020, at https://doi.org/10.1016/j.oregeorev.2016.10.033.

Cannon, W.F., Kimball, B.E., and Corathers, L.A., 2017, Manganese, chap. L of Schulz, K.J., DeYoung, J.H., Jr., Seal, R.R., II, and Bradley, D.C., eds., Critical mineral resources of the United States-Economic and environmental geology and prospects for future supply: U.S. Geological Survey Professional Paper 1802, p. L1-L28, accessed April 18, 2020, at https://doi.org/10.3133/pp1802L.

Černý, P., and Ercit, T.S., 2005, The classification of granitic pegmatites revisited: Canadian Mineralogist, v. 43, no. 6, p. 2005-2026, accessed April 18, 2020, at https://doi.org/ 10.2113/gscanmin.43.6.2005. 
Cox, D.P., and Singer, D.A., 2007, Descriptive and gradetonnage models and database for iron oxide $\mathrm{Cu}-\mathrm{Au}$ deposits: U.S. Geological Survey Open-File Report 2007-1155, 13 p., accessed April 18, 2020, at https://doi.org/10.3133/ ofr20071155.

Craddock, W.H., Blondes, M.S., DeVera, C.A., and Hunt, A.G., 2017, Mantle and crustal gases of the Colorado Plateau - Geochemistry, sources, and migration pathways: Geochimica et Cosmochimica Acta, v. 213, p. 346-374.

Day, W.C., 2019, The Earth Mapping Resources Initiative (Earth MRI) - Mapping the Nation's critical mineral resources (ver. 1.2, September 2019): U.S. Geological Survey Fact Sheet 2019-3007, 2 p., accessed April 18, 2020, at https://doi.org/10.3133/fs20193007.

Denny, F.B., Devera, J.A., and Seid, M.J., 2016, Fluorite deposits within the Illinois-Kentucky Fluorspar District and how they relate to the Hicks Dome cryptoexplosive feature, Hardin County, Illinois, in Lasemi, Z., and Elrick, S., eds., 1967-2016-Celebrating 50 years of geoscience in the mid-continent, Guidebook for the 50th Annual Meeting of the Geological Society of America North-Central Section, April 18-19, 2016: Illinois State Geological Survey Guidebook 43, p. 39-54.

Denny, F.B., Guillemette, R.N., and Lefticariu, L., 2015, Rare earth mineral concentrations in ultramafic alkaline rocks and fluorite within the Illinois-Kentucky Fluorite District-Hicks Dome cryptoexplosive complex, southeast Illinois and northwest Kentucky (USA), in Lasemi, Z., ed., Proceedings of the 47th Forum on the Geology of Industrial Minerals: Illinois State Geological Survey Circular 587, p. $77-92$.

Dicken, C.L., Horton, J.D., San Juan, C.A., Anderson, A.K., Ayuso, R.A., Bern, C.R., Bookstrom, A.A., Bradley, D.C., Bultman, M.W., Carter, M.W., Cossette, P.M., Day, W.C., Drenth, B.J., Emsbo, P., Foley, N.K., Frost, T.P., Gettings, M.E., Hammarstrom, J.M., Hayes, T.S., Hofstra, A.H., Hubbard, B.E., John, D.A., Jones, J.V., III, Kreiner, D.C., Lund, K., McCafferty, A.E., Merchat, A.J., Ponce, D.A., Schulz, K.J., Shah, A.K., Siler, D.L., Taylor, R.D., Vikre, P.G., Walsh, G.J., Woodruff, L.G., and Zurcher, L., 2019, GIS and data tables for focus areas for potential domestic nonfuel sources of rare earth elements: U.S. Geological Survey data release, accessed April 18, 2020, at https://doi.org/10.5066/P95CHIL0.

Dostal, J., 2016, Rare metal deposits associated with alkaline/peralkaline igneous rocks, chap. 2 of Verplanck, P.L., and Hitzman, M.W., eds., Reviews in economic geology, volume 18-Rare earth and critical elements in ore deposits: Littleton, Colo., Society of Economic Geologists, Inc., p. 33-54.
Dostal, J., 2017, Rare earth element deposits of alkaline igneous rocks: Resources, v. 6, no. 3, article no. 34, 12 p., accessed March 30, 2021, at https://www.mdpi.com/20799276/6/3/34.

DSM Observer, 2020, Beyond batteries-Exploring the demand for scandium and tellurium from the deep ocean: Deep Sea Mining News \& Resources, November 19, 2020, accessed April 12, 2021, at http://dsmobserver.com/2020/11/ beyond-batteries-exploring-the-demand-for-scandium-andtellurium-from-the-deep-ocean/.

Dyni, J.R., 1991, Descriptive model of sodium carbonate in bedded lacustrine evaporites-Deposit subtype - Green River (Model 35ba), in Orris, G.J., and Bliss, J.D., eds., Some industrial mineral deposit models-Descriptive deposit models: U.S. Geological Survey Open-File Report 91-11A, p. 46-50.

Emsbo, P., 2000, Gold in sedex deposits, in Hagemann, S.G., and Brown, P.E., eds., Reviews in economic geology, volume 13-Gold in 2000: Littleton, Colo., Society of Economic Geologists, Inc., p. 427-437.

Emsbo, P., 2009, Geologic criteria for the assessment of sedimentary exhalative (sedex) $\mathrm{Zn}-\mathrm{Pb}-\mathrm{Ag}$ deposits: U.S. Geological Survey Open-File Report 1209, 21 p. [Also available at https://doi.org/10.3133/ofr20091209.]

Emsbo, P., McLaughlin, P.I., Breit, G.N., du Bray, E.A., and Koenig, A.E., 2015, Rare earth elements in sedimentary phosphate deposits - Solution to the global REE crisis?: Gondwana Research, v. 27, p. 776-785, accessed April 18, 2020, at https://doi.org/10.1016/j.gr.2014.10.008.

Emsbo, P., McLaughlin, P.I., du Bray, E.A., Anderson, E.D., Vandenbroucke, T.R.A., and Zielinski, R.A., 2016b, Rare earth elements in sedimentary phosphorite deposits-A global assessment, chap. 5 of Verplanck, P.L, and Hitzman, M.W., eds., Reviews in economic geology, volume 18Rare earth and critical elements in ore deposits: Littleton, Colo., Society of Economic Geologists, Inc., p. 101-114.

Emsbo, P., Seal, R.R., Breit, G.N., Diehl, S.F., and Shah, A.K., 2016a, Sedimentary exhalative (sedex) zinc-leadsilver deposit model: U.S. Geological Survey Scientific Investigations Report 2010-5070-N, 57 p., accessed April 18, 2020, at https://dx.doi.org/10.3133/sir20105070N.

Ernst, R.E., and Jowitt, S.M., 2013, Large igneous provinces (LIPs) and metallogeny, chap. 2 of Colpron, M., Bissing, T., Rusk, B.G., and Thompson, J.F.H., eds., Tectonics, metallogeny, and discovery - The North American Cordillera and similar accretionary settings: Tulsa, Okla., Society of Economic Geologists, Special Publications, volume 17, p. 17-51. 
Executive Office of the President, 2017, A Federal strategy to ensure secure and reliable supplies of critical minerals: Federal Register, v. 82, no. 246, p. 60835-60837, accessed April 18, 2020, at https://www.federalregister.gov/ documents/2017/12/26/2017-27899/a-federal-strategy-toensure-secure-and-reliable-supplies-of-critical-minerals.

Foley, N.K., and Ayuso, R.A., 2015, REE enrichment in granite-derived regolith deposits of the southeast United States-Prospective source rocks and accumulation processes, in Simandl, G.J., and Neetz, M., eds., Symposium on strategic and critical materials proceedings: British Columbia Geological Survey Paper 2015-3, p. 131-138.

Foley, N.K., Hofstra, A.H., Lindsey, D.A., Seal, R.R., II, Jaskula, B., and Piatak, N.M., 2012, Occurrence model for volcanogenic beryllium deposits, chap. F of Mineral deposit models for resource assessment: U.S. Geological Survey Scientific Investigations Report 2010-5070-F, 43 p., accessed April 18, 2020, at https://pubs.usgs.gov/sir/2010/ 5070/f/SIR10-5070F.pdf.

Force, E.R., Paradis, S., and Simandl, G.J., 1999, Sedimentary manganese, in Simandl, G.J., Hora, Z.D., and Lefebure D.V., eds., Selected British Columbia mineral deposit profiles, volume 3-Industrial minerals: British Columbia Ministry of Energy and Mines, Open File 1999-10 p. $47-50$.

Fortier, S.M., Nassar, N.T., Lederer, G.W., Brainard, J., Gambogi, J., and McCullough, E.A., 2018, Draft critical mineral list - Summary of methodology and background information-U.S. Geological Survey technical input document in response to Secretarial Order No. 3359: U.S. Geological Survey Open-File Report 2018-1021, 15 p., accessed April 18, 2020, at https://doi.org/10.3133/ ofr20181021.

Geological Survey of Western Australia, 2019, Mineral Systems Atlas: Government of Western Australia, Department of Mines, Industry Regulation and Safety website, accessed April 18, 2020, at http://www.dmp.wa.gov.au/msa.

Geoscience Australia, 2019, Mineral Systems of Australia, accessed April 18, 2020, at https://www.ga.gov.au/about/ projects/resources/mineral-systems.

Goldfarb, R.J., Baker, T., Dubé, B., Groves, D.I., Hart, C.J.R., and Gosselin, P., 2005, Distribution, character, and genesis of gold deposits in metamorphic terranes: Littleton, Colo., Society of Economic Geologists, Inc., Economic Geology 100th Anniversary Volume, p. 407-450.
Goldfarb, R.J., Hofstra, A.H., and Simmons, S.F., 2016, Critical elements in Carlin, epithermal, and orogenic gold deposits, chap. 10 of Verplanck, P.L., and Hitzman, M.W., eds., Reviews in economic geology, volume 18-Rare earth and critical elements in ore deposits: Littleton, Colo., Society of Economic Geologists, Inc., p. 217-244. [Also available at https://doi.org/10.5382/Rev.18.10.]

Gray, J.E., and Bailey, E.A., 2003, The southwestern Alaska mercury belt, in Gray, J.E., ed., Geologic studies of mercury by the U.S. Geological Survey: U.S. Geological Survey Circular 1248, p. 19-22.

Groves, D.I., Bierlein, F.P., Meinert, L.D., and Hitzman, M.W., 2010, Iron oxide copper-gold (IOCG) deposits through Earth history-Implications for origin, lithospheric setting, and distinction from other epigenetic iron oxide deposits: Economic Geology, v. 105, no. 3, p. 641-654, accessed April 18, 2020, at https://doi.org/10.2113/ gsecongeo.105.3.641.

Groves, D.I., Goldfarb, R.J., Gebre-Mariam, M., Hagemann, S.G., and Robert, F., 1998, Orogenic gold deposits-A proposed classification in the context of their crustal distribution and relationship to other gold deposit types: Ore Geology Reviews, v. 13, no. 1-5, p. 7-27. [Also available at https://doi.org/10.1016/S0169-1368(97)00012-7.]

Hall, S.M., Van Gosen, B.S., Paces, J.B., Zielinski, R.A., and Breit, G.N., 2019, Calcrete uranium deposits in the Southern High Plains, USA: Ore Geology Reviews, v. 109, p. 50-78, accessed April 18, 2020, at https://doi.org/ 10.1016/j.oregeorev.2019.03.036.

Hammarstrom, J.H., and Dicken, C.L., 2019, Focus areas for data acquisition for potential domestic sources of critical minerals - Rare earth elements, chap. A of U.S. Geological Survey, Focus areas for data acquisition for potential domestic sources of critical minerals: U.S. Geological Survey Open-File Report 2019-1023, 11 p., accessed April 18, 2020, at https://doi.org/10.3133/ofr20191023A.

Hart, C.J.R., 2007, Reduced intrusion-related gold systems, in Goodfellow, W.D., ed., Mineral deposits of Canada-A synthesis of principal deposit types, district metallogeny, the evolution of geological provinces, and exploration methods: Geological Association of Canada, Mineral Deposits Division, Special Publication 5, p. 95-112.

Hayes, S.M., and McCullough, E.A., 2018, Critical minerals - A review of elemental trends in comprehensive criticality studies: Resources Policy, v. 59, p. 192-199. [Also available at https://doi.org/10.1016/ j.resourpol.2018.06.015.] 
Hayes, T.S., Cox, D.P., Piatak, N.M., and Seal, R.R., II, 2015, Sediment-hosted stratabound copper deposit model: U.S. Geological Survey Scientific Investigations Report 2010-5070-M, 147 p., accessed April 18, 2020, at https://doi.org/10.3133/sir20105070M.

Hayes, T.S., Miller, M.M., Orris, G.J., and Piatak, N.M., 2017, Fluorine, chap. G of Schulz, K.J., DeYoung, J.H., Jr., Seal, R.R., II, and Bradley, D.C., eds., Critical mineral resources of the United States-Economic and environmental geology and prospects for future supply: U.S. Geological Survey Professional Paper 1802, p. G1-G80, accessed April 18, 2020, at https://doi.org/10.3133/pp1802G.

Hofstra, A.H., and Cline, J.S., 2000, Characteristics and models for Carlin-type gold deposits, chap. 5 of Hagemann, S.G., and Brown, P.E., eds., Reviews in economic geology, volume 13-Gold in 2000: Littleton, Colo., Society of Economic Geologists, Inc., p. 163-220.

Hofstra, A.H., Cosca, M.A., and Rockwell, B.W., 2014, Advanced argillic lithocaps above Climax-type Mo porphyries? Evidence from porphyry clusters in New Mexico, Utah, and Colorado: Society of Economic Geologists Annual Meeting, Keystone, Colorado, 1 p.

Hofstra, A.H., Marsh, E.E., Todorov, T.I., and Emsbo, P., 2013a, Fluid inclusion evidence for a genetic link between simple antimony veins and giant silver veins in the Coeur d'Alene mining district, ID and MT, U.S.A: Geofluids, v. 13, no. 4, p. 475-493, accessed April 18, 2020, at https://doi.org/10.1111/gfl.12036.

Hofstra, A.H., Todorov, T.I., Mercer, C.N., Adams, D.T., and Marsh, E.E., 2013b, Silicate melt inclusion evidence for extreme pre-eruptive enrichment and post-eruptive depletion of lithium in silicic volcanic rocks of the western United States-Implications for the origin of lithium-rich brines: Economic Geology, v. 108, no. 7, p. 1691-1701, accessed April 18, 2020, at https://doi.org/10.2113/ econgeo.108.7.1691.

Horn, C.M., Keeling, J.L., and Olliver, J.G., 2017, Sedimentary magnesite deposits, Flinders Range, in Phillips, N., ed., 2017, Australian ore deposits: The Australasian Institute of Mining and Metallurgy, Monograph 32, p. 671-672.

Hulsbosch, N., 2019, Nb-Ta-Sn-W distribution in graniterelated ore systems-Fractionation mechanisms and examples from the Karagwe-Ankole Belt of Central Africa, chap. 4 of Decrée, S., and Rob, 1., eds., Ore deposits - Origin, exploration, and exploitation: American Geophysical Union, Geophysical Monograph 242, p. $75-107$.

Hunt, J.M., ed., 1996, Petroleum geochemistry and geology ( $2 \mathrm{~d}$ ed.): New York, W.H. Freeman, 743 p.
Huston, D.L., Mernagh, T.P., Hagemann, S.G., Doublier, M.P., Fiorentini, M., Champion, D.C., Jaques, A.L., Czarnota, K., Cayley, R., Skirrow, R., and Bastrakov, E., 2016, Tectonometallogenic systems - The place of mineral systems within tectonic evolution, with an emphasis on Australian examples: Ore Geology Reviews, v. 76, p. 168-210. [Also available at https://doi.org/10.1016/j.oregeorev.2015.09.005.]

International Atomic Energy Agency [IAEA], 2020, Descriptive uranium deposit and mineral system models: Vienna, Austria, International Atomic Energy Agency, 313 p.

Jensen, E.P., and Barton, M.D., 2000, Gold deposits related to alkaline magmatism, chap. 8 of Hagemann, S.G., and Brown, P.E., eds., Reviews in economic geology, volume 13-Gold in 2000: Littleton, Colo., Society of Economic Geologists, Inc., p. 279-314.

John, D.A., Ayuso, R.A., Barton, M.D., Blakely, R.J., Bodnar, R.J., Dilles, J.H., Gray, F., Graybeal, F.T., Mars, J.C., McPhee, D.K., Seal, R.R., Taylor, R.D., and Vikre, P.G., 2010, Porphyry copper deposit model, chap. B of Mineral deposit models for resource assessment: U.S. Geological Survey Scientific Investigations Report 2010-5070-B, 169 p., accessed April 18, 2020, at https://pubs.usgs.gov/sir/ 2010/5070/b/pdf/SIR10-5070B.pdf.

John, D.A., and Henry, C.H., 2020, Magmatic-tectonic settings of Cenozoic epithermal gold-silver deposits of the Great Basin, Western United States, in Geological Society of Nevada Symposium, 2020, Sparks, Nev., May 18-21, 2020, Proceedings: Reno, Nev., Geological Society of Nevada, p. 765-796.

John, D.A., Seal, R.R., II, and Polyak, D.E., 2017, Rhenium, chap. P of Schulz, K.J., DeYoung, J.H., Jr., Seal, R.R., II, and Bradley, D.C., eds., Critical mineral resources of the United States-Economic and environmental geology and prospects for future supply: U.S. Geological Survey Professional Paper 1802, p. P1-P49, accessed April 18, 2020, at https://doi.org/10.3133/pp1802P.

John, D.A., and Taylor, R.D., 2016, Byproducts of porphry copper and molybdenum deposits, chap. 8 of Verplanck, P.L, and Hitzman, M.W., eds., Reviews in economic geology, volume 18 - Rare earth and critical elements in ore deposits: Littleton, Colo., Society of Economic Geologists, Inc., p. 137-164.

Johnson, C.A., Piatak, N.M., and Miller, M.M., 2017, Barite (barium), chap. D of Schulz, K.J., DeYoung, J.H., Jr., Seal, R.R., II, and Bradley, D.C., eds., Critical mineral resources of the United States-Economic and environmental geology and prospects for future supply: U.S. Geological Survey Professional Paper 1802, p. D1-D18, accessed April 18, 2020, at https://doi.org/10.3133/pp1802D. 
Jones, J.V., III, Piatak, N.M., and Bedinger, G.M., 2017 , Zirconium and hafnium, chap. V of Schulz, K.J., DeYoung, J.H., Jr., Seal, R.R., II, and Bradley, D.C., eds., Critical mineral resources of the United States-Economic and environmental geology and prospects for future supply: U.S. Geological Survey Professional Paper 1802, p. V1V26, accessed April 18, 2020, at https://doi.org/10.3133/ pp1802V.

Kamilli, R.J., Kimball, B.E., and Carlin, J.F., Jr., 2017, Tin, chap. S of Schulz, K.J., DeYoung, J.H., Jr., Seal, R.R., II, and Bradley, D.C., eds., Critical mineral resources of the United States - Economic and environmental geology and prospects for future supply: U.S. Geological Survey Professional Paper 1802, p. S1-S53, accessed April 18, 2020, at https://doi.org/10.3133/pp1802S.

Kelley, K.D., and Spry, P.G., 2016, Critical elements in alkaline igneous rock-related epithermal gold deposits, chap. 9 of Verplanck, P.L, and Hitzman, M.W., eds., Reviews in economic geology, volume 18-Rare earth and critical elements in ore deposits: Littleton, Colo., Society of Economic Geologists, Inc., p. 195-216.

King, H.M., 2020, Helium-A byproduct of the natural gas industry: Geology.com web page, accessed October 7, 2020, at https://geology.com/articles/helium/.

Kissin, S.A., 1992, Five-element (Ni-Co-As-Ag-Bi) veins: Geoscience Canada, v. 19, p. 113-124.

Knox-Robinson, C.M., and Wyborn, L.A.I., 1997, Towards a holistic exploration strategy-Using Geographic Information Systems as a tool to enhance exploration: Australian Journal of Earth Sciences, v. 44, no. 4, p. 453-463. [Also available at https://doi.org/10.1080/ 08120099708728326.]

Leach, D.L., Hofstra, A.H., Church, S.E., Snee, L.W., Vaughn, R.B., and Zartman, R.E., 1998, Evidence for Proterozoic and Late Cretaceous-early Tertiary ore-forming events in the Coeur d'Alene district, Idaho and Montana: Economic Geology, v. 93, no. 3, p. 347-359. [Also available at https://doi.org/10.2113/gsecongeo.93.3.347.]

Leach, D.L., Landis, G.P., and Hofstra, A.H., 1988, Metamorphic origin of the Coeur d'Alene base- and precious-metal veins in the Belt basin, Idaho and Montana, v. 16, p. 122-125. [Also available at https://doi.org/10.1130/ 0091-7613(1988)016<0122:MOOTCD>2.3.CO;2.]

Leach, D.L., Taylor, R.D., Fey, D.L., Diehl, S.F., and Saltus, R.W., 2010, A deposit model for Mississippi Valley-Type lead-zinc ores, chap. A of Mineral deposit models for resource assessment: U.S. Geological Survey Scientific Investigations Report 2010-5070-A, 52 p., accessed April 18, 2020, at https://pubs.usgs.gov/sir/2010/5070/a/ pdf/SIR10-5070A.pdf.
Lefebure, D.V., and Coveney, R.M., Jr., 1995, Shale-hosted Ni-Zn-Mo-PGE, in Lefebure, D.V., and Ray, G.E., eds., Selected British Columbia mineral deposit profiles, volume 1-Metallics and coal: British Columbia Ministry of Energy of Employment and Investment, Open File 1995-20, p. $45-48$.

Levson, V.M., 1995, Marine placers, in Lefebure D.V., and Ray G.E., eds., Selected British Columbia mineral deposit profiles, volume 1-Metallics and coal: British Columbia Ministry of Energy of Employment and Investment, Open File 1995-20, p. 29-31.

London, D., 2008, Pegmatites: The Canadian Mineralogist, Special Publication 10, 347 p.

London, D., 2016, Rare-element granitic pegmatites, chap. 8 of Verplanck, P.L, and Hitzman, M.W., eds., Reviews in economic geology, volume 18-Rare earth and critical elements in ore deposits: Littleton, Colo., Society of Economic Geologists, Inc., p. 165-194.

Long, K.R., Wynn, J.C., Fritz, F., and Corbett, J., 1992, Lacustrine manganese-A supplement to U.S. Geological Survey Bulletin 1693: U.S. Geological Survey Open-File Report 92-239, $12 \mathrm{p}$.

Ludington, S., and Plumlee, G.S., 2009, Climax-type porphyry molybdenum deposits: U.S. Geological Survey Open-File Report 2009-1215, 16 p.

Luque, F.J., Huizenga, J.M., Crespo-Feo, E., Wada, H., Ortega, L., and Barrenechea, J.F., 2014, Vein graphite deposits - Geological settings, origin, and economic significance: Mineralium Deposita, v. 49, no. 2, p. 261-277. [Also available at https://doi.org/10.1007/s00126-013-0489-9.]

Magoon, L.B., and W.G. Dow, eds., 1994, The petroleum system-From source to trap: Tulsa, Okla., American Association of Petroleum Geologists, AAPG Memoir 60, 620 p.

Manning, A.H., and Emsbo, P., 2018, Testing the potential role of brine reflux in the formation of sedimentary exhalative (sedex) ore deposits: Ore Geology Reviews, v. 102, p. 862-874. [Also available at https://doi.org/10.1016/ j.oregeorev.2018.10.003.]

Markl, G., Burisch, M., and Neumann, U., 2016, Natural fracking and the genesis of five-element veins: Mineralium Deposita, v. 51, no. 6, p. 703-712. [Also available at https://doi.org/10.1007/s00126-016-0662-z.]

Marsh, E., Anderson, E., and Gray, F., 2013, Nickel-cobalt laterites-A deposit model, chap. H of Mineral deposit models for resource assessment: U.S. Geological Survey Scientific Investigations Report 2010-5070-H, 38 p., accessed April 18, 2020, at https://pubs.usgs.gov/sir/ 2010/5070/h/. 
Marsh, E.E., Hitzman, M.W., and Leach, D.L., 2016, Critical elements in sediment-hosted deposits (clastic-dominated $\mathrm{Zn}-\mathrm{Pb}-\mathrm{Ag}$, Mississippi Valley-type $\mathrm{Zn}-\mathrm{Pb}$, sedimentary rock-hosted stratiform $\mathrm{Cu}$, and carbonate-hosted polymetallic deposits)-A review, chap. 12 of Verplanck, P.L and Hitzman, M.W., eds., Reviews in economic geology, volume 18-Rare earth and critical elements in ore deposits: Littleton, Colo., Society of Economic Geologists, Inc., p. 307-322.

Martin, R.F., and De Vito, C., 2005, The patterns of enrichment in felsic pegmatites ultimately depend on tectonic setting: Canadian Mineralogist, v. 43, no. 6 , p. 2027-2048. [Also available at https://doi.org/10.2113/ gscanmin.43.6.2027.]

McCuaig, T.C., Beresford, S., and Hronsky, J., 2010, Translating the mineral systems approach into an effective exploration targeting system: Ore Geology Reviews, v. 38, no. 3, p. 128-138. [Also available at https://doi.org/ 10.1016/j.oregeorev.2010.05.008.]

McKinney, S.T., Cottle, J.M., and Lederer, G.W., 2015, Evaluating rare earth element (REE) mineralization mechanisms in Proterozoic gneiss, Music Valley, California: Geological Society of America Bulletin, v. 127, p. 1135-1152. [Also available at https://doi.org/10.1130/ B31165.1.]

Menzel, M.D., Garrido, C.J., Sánchez-Vizcaíno, V.L., Marchesi, C., Hidas, K., Escayola, M.P., and Huertas, A.D., 2018, Carbonation of mantle peridotite by CO2-rich fluids-The formation of listvenites in the Advocate ophiolite complex (Newfoundland, Canada): Lithos, v. 323, p. 238261, accessed March 30, 2021, at https://doi.org/10.1016/j. lithos.2018.06.001.

Mondal, S.K., and Griffin, W.L., eds., 2018, Processes and ore deposits of ultramafic-mafic magmas through space and time: Amsterdam, Elsevier, 364, p.

Monecke, T., Petersen, S., Hannington, M.D., Grant, H., and Samson, I.M., 2016, The minor element endowment of modern sea-floor massive sulfides and comparison with deposits hosted in ancient volcanic successions, chap. 11 of Verplanck, P.L., and Hitzman, M.W., eds., Reviews in economic geology, volume 18 - Rare earth and critical elements in ore deposits: Littleton, Colo., Society of Economic Geologists, Inc., p. 245-306. [Also available at https://doi.org/10.5382/ Rev.18.11.]

Mountney, N.P., 2005, Sedimentary environments/deserts, in Encyclopedia of Geology, p. 539-549, accessed March 30, 2021, at https://doi.org/10.1016/B0-12-369396-9/00176-3.
Munk, L., Hynek, S.A., Bradley, D.C., Boutt, D., Labay, K., and Jochens, H., 2016, Lithium brines-A global perspective, chap. 14 of Verplanck, P.L, and Hitzman, M.W., eds., Reviews in economic geology, volume 18-Rare earth and critical elements in ore deposits: Littleton, Colo., Society of Economic Geologists, Inc., p. 339-365.

Muntean, J.L., 2018, The Carlin gold system-Application to exploration in Nevada and beyond, chap. 2 of Muntean, J.L., ed., Reviews in economic geology, volume 20Diversity of Carlin-style gold deposits: Littleton, Colo., Society of Economic Geologists, Inc., p. 39-88. [Also available at https://doi.org/10.5382/rev.20.02.]

Nutt, C.J., and Hofstra, A.H., 2007, Bald Mountain gold mining district, Nevada-A Jurassic reduced intrusionrelated gold system: Economic Geology, v. 102, no. 6, p. 1129-1155. [Also available at https://doi.org/10.2113/ gsecongeo.102.6.1129.]

Orris, G.J., 1995, Borate deposits: U.S. Geological Survey Open-File Report 95-842, 57 p.

Otton, J.K., Bradbury, J.P., Forester, R.M., and Hanley, J., 1990, Paleontological analysis of a lacustrine carbonaceous uranium deposit at the Anderson mine, Date Creek basin, west-central Arizona (U.S.A): Ore Geology Reviews, v. 5, p, 541-552.

Panteleyev, A., 1996, Sn-Ag veins, in Lefebure, D.V. and Hõy, T., eds., Selected British Columbia mineral deposit profiles, volume 2-Metallic deposits: British Columbia Ministry of Employment and Investment, Open File 1996-13, p. 45-48.

Plumlee, G.S., Goldhaber, M.B., and Rowan, E.L., 1995, The potential role of magmatic gases in the genesis of IllinoisKentucky fluorspar deposits-Implications from chemical reaction path modeling: Economic Geology, v. 90, no. 5, p. 999-1011. [Also available at https://doi.org/10.2113/ gsecongeo.90.5.999.]

Power, 1.M., Harrison, A.L., Dipple, G.M., Wilson, S.A., Barker, S.L.L., and Fallone, S.J., 2019, Magnesite forma-tion in playa environments near Atlin, British Columbia, Canada: Geochimica et Cosmochimica Acta, v. 255, p. 1-24, accessed March 30, 2021, at https:// doi.org/10.1016/j. gca.2019.04.008.

Raup, O.B., 1991a, Descriptive model of bedded saltDeposit subtype-Marine evaporite salt (Model 35ac), in Orris, G.J., and Bliss, J.D., eds., Some industrial mineral deposit models - Descriptive deposit models: U.S. Geological Survey Open-File Report 91-11A, p. 33-35.

Raup, O.B., 1991b, Descriptive model of bedded gypsumDeposit subtype-Marine evaporite gypsum (Model 35ae), in Orris, G.J., and Bliss, J.D., eds., Some industrial mineral deposit models—Descriptive deposit models: U.S. Geological Survey Open-File Report 91-11A, p. 39-41. 
Robinson, G.R., Jr., Hammarstrom, J.M., and Olson, D.W., 2017, Graphite, chap. J of Schulz, K.J., DeYoung, J.H., Jr., Seal, R.R., II, and Bradley, D.C., eds., Critical mineral resources of the United States-Economic and environmental geology and prospects for future supply: U.S. Geological Survey Professional Paper 1802, p. J1-J24, accessed April 18, 2020, at https://doi.org/10.3133/pp1802J.

Sanematsu, K., and Watanabe, Y., 2016, Characteristics and genesis of ion adsorption-type rare earth element deposits, chap. 3 of Verplanck, P.L., and Hitzman, M.W., eds., Reviews in economic geology, volume 18-Rare earth and critical elements in ore deposits: Littleton, Colo., Society of Economic Geologists, Inc., p. 55-80. [Also available at https://doi.org/10.5382/Rev.18.03.]

Scharrer, M., Kreissl, S., and Markl, G., 2019, The mineralogical variability of hydrothermal native element-arsenide (five-element) associations and the role of physicochemical and kinetic factors concerning sulfur and arsenic: Ore Geology Reviews, v. 113, p. 103025, accessed April 18, 2020, at https://doi.org/10.1016/j.oregeorev.2019.103025.

Schulte, R.F., Taylor, R.D., Piatak, N.M., and Seal, R.R., II, 2012, Stratiform chromite deposit model, chap. E of Mineral deposit models for resource assessment: U.S. Geological Survey Scientific Investigations Report 2010-5070-E, 131 p.

Seal, R.R., II, Schulz, K.J., and DeYoung, J.H., Jr., with contributions from David M. Sutphin, Lawrence J. Drew, James F. Carlin, Jr., and Byron R. Berger, 2017, Antimony, chap. C of Schulz, K.J., DeYoung, J.H., Jr., Seal, R.R., II, and Bradley, D.C., eds., Critical mineral resources of the United States-Economic and environmental geology and prospects for future supply: U.S. Geological Survey Professional Paper 1802, p. C1-C17, accessed April 18, 2020, at https://doi.org/10.3133/pp1802C.

Seedorff, E., Dilles, J.H., Proffett, J.M., Jr., Einaudi, M.T., Zurcher, L., Stavast, W.J.A., Johnson, D.A., and Barton, M.D., 2005, Porphyry deposits-Characteristics and origin of hypogene features, chap. 10 of Hedenquist, J.W., Thompson, J.F.H., Goldfarb, R.J., and Richards, J.P., Economic Geology 100th Anniversary Volume: Littleton, Colo., Society of Economic Geologists, Inc., p. 251-298, accessed April 18, 2020, at https://doi.org/10.5382/ AV100.10.

Sengupta, D., and Van Gosen, B.S., 2016, Placer-type rare earth element deposits, chap. 4 of Verplanck, P.L., and Hitzman, M.W., eds., Reviews in economic geology, volume 18-Rare earth and critical elements in ore deposits: Littleton, Colo., Society of Economic Geologists, Inc., p. $81-100$.
Shanks, W.C., III, and Thurston, R., 2012, Volcanogenic massive sulfide occurrence model: U.S. Geological Survey Scientific Investigations Report 2010-5070-C, 345 p., accessed April 18, 2020, at https://pubs.usgs.gov/sir/2010/ 5070/c/SIR10-5070-C.pdf.

Sheppard, R.A., 1991a, Descriptive model of sedimentary zeolites-Deposit subtype-Zeolites in tuffs of open hydrologic systems (Model 25oa), in Orris, G.J., and Bliss, J.D., eds., Some industrial mineral deposit models-Descriptive deposit models: U.S. Geological Survey Open-File Report 91-11A, p. 16-18.

Sheppard, R.A., 1991b, Descriptive model of sedimentary zeolites-Deposit subtype-Zeolites in tuffs of saline, alkaline-lake deposits (Model 25ob), in Orris, G.J., and Bliss, J.D., eds., Some industrial mineral deposit modelsDescriptive deposit models: U.S. Geological Survey OpenFile Report 91-11A, p. 19-21.

Sillitoe, R.H., 2010, Porphyry copper systems: Economic Geology, v. 105, no. 1, p. 3-41. [Also available at https://doi.org/10.2113/gsecongeo.105.1.3.]

Sillitoe, R.H., Steele, G.B., Thompson, J.F.H., and Lang, J.R., 1998, Advanced argillic lithocaps in the Bolivian tin-silver belt: Mineralium Deposita, v. 33, no. 6, p. 539-546. [Also available at https://doi.org/10.1007/s001260050170.]

Simmons, S.F., White, N.C, and John, D.A., 2005, Geological characteristics of epithermal precious and base metal deposits, in Hedenquist, J.W., Thompson, J.F.H., Goldfarb, R.J., and Richards, J.P., eds., Economic Geology-One Hundredth Anniversary Volume, 1905-2005: Littleton, Colo., Society of Economic Geologists, Inc., p. 485-522, accessed March 30, 2021, at https://doi.org/10.5382/AV100.16.

Skirrow, R.G., Jaireth, S., Huston, D.L., Bastrakov, E.N., Schofield, A., van der Wielen, S.E., and Barnicoat, A.C., 2009, Uranium mineral systems - Processes, exploration criteria and a new deposit framework: Geoscience Australia, Geoscience Australia Record 2009/20, 44 p.

Slack, J.F., ed., 2013, Descriptive and geoenvironmental model for cobalt-copper-gold deposits in metasedimentary rocks (ver. 1.1, March 14, 2014): U.S. Geological Survey Scientific Investigations Report 2010-5070-G, 218 p., accessed April 18, 2020, at https://doi.org/10.3133/ sir20105070G.

Slack, J.F., Corriveau, L., and Hitzman, M.W., 2016, A special issue devoted to Proterozoic iron oxide-apatite $( \pm \mathrm{REE})$ and iron oxide copper-gold and affiliated deposits of southeast Missouri, USA, and the Great Bear magmatic zone, Northwest Territories, Canada-Preface: Economic Geology, v. 111, no. 8, p. 1803-1814. [Also available at https://doi.org/10.2113/econgeo.111.8.1803.] 
Sloan, R.E., 1964, The Cretaceous system in Minnesota: Minnesota Geological Survey, Report of Investigations 5, 64 p.

Sutherland, W.M., and Cola, E.C., 2016, A comprehensive report on rare earth elements in Wyoming: Laramie, Wyo., Wyoming State Geological Survey Report of Investigations $71,137 \mathrm{p}$.

Sutphin, D.M., 1991a, Descriptive model of amorphous graphite (Model 18k), in Orris, G.J., and Bliss, J.D., eds., Some industrial mineral deposit models-Descriptive deposit models: U.S. Geological Survey Open-File Report 91-11A, p. 9-10.

Sutphin, D.M., 1991b, Descriptive model of disseminated flake graphite (Model 37f), in Orris, G.J., and Bliss, J.D., eds., Some industrial mineral deposit models-Descriptive deposit models: U.S. Geological Survey Open-File Report 91-11A, p. 55-57.

Sutphin, D.M., 1991c, Descriptive model of graphite veins (Model 37g), in Orris, G.J., and Bliss, J.D., eds., Some industrial mineral deposit models-Descriptive deposit models: U.S. Geological Survey Open-File Report 91-11A, p. 58-60.

Taylor, R.D., Hammarstrom, J.M., Piatak, N.M., and Seal, R.R., II, 2012, Arc-related porphyry molybdenum deposit model, chap. D of Mineral deposit models for resource assessment: U.S. Geological Survey Scientific Investigations Report 2010-5070-D, 64 p.

Tosdal, R., Dilles, J.H., and Cooke, D.R., 2009, From source to sinks in auriferous magmatic-hydrothermal porphyry and epithermal deposits: Elements, v. 5, no. 5, p. 289-295, accessed April 18, 2020, at https://doi.org/10.2113/ gselements.5.5.289.

U.S. Department of the Interior, 2017, Critical mineral independence and security: U.S. Department of the Interior, Order No. 3359, accessed April 18, 2020, at https://www.doi.gov/ sites/doi.gov/files/uploads/so_criticalminerals.pdf.

Van Gosen, B.S., Fey, D.L., Shah, A.K., Verplanck, P.L., and Hoefen, T.M., 2014, Deposit model for heavy-mineral sands in coastal environments: U.S. Geological Survey Scientific Investigations Report 2010-5070-L, 51 p., accessed April 18, 2020, at https://doi.org/10.3133/sir20105070L.

Verplanck, P.L., Mariano, A.N., and Mariano, A., Jr., 2016, Rare earth elements in carbonatites, chap. 1 of Verplanck, P.L., and Hitzman, M.W., eds., Reviews in economic geology, volume 18-Rare earth and critical elements in ore deposits: Littleton, Colo., Society of Economic Geologists, Inc., p. 5-32.

Verplanck, P.L., Van Gosen, B.S., Seal, R.R., and McCafferty, A.E., 2014, A deposit model for carbonatite and peralkaline intrusion-related rare earth element deposits: U.S. Geological Survey Scientific Investigations Report 2010-5070-J, 58 p., accessed April 18, 2020, at https://doi.org/10.3133/sir20105070J.
Wallace, A.R., and Whelan, J.F., 1986, The Schwartzwalder uranium deposit-III, Alteration, vein mineralization, light stable isotopes, and genesis of the deposit: Economic Geology, v. 81, no. 4, p. 872-888.

Wang, Z., Li, M.Y.H., Liu, Z.R.R., and Zhou, M.F., 2021, Scandium - Ore deposits, the pivotal role of magmatic enrichment and future exploration: Ore Geology Reviews, v. 128, article no. 103906, accessed April 12, 2021, at https://doi.org/10.1016/j.oregeorev.2020.103906.

Warren, J.K., 2010, Evaporites through time-Tectonic, climatic and eustatic controls in marine and nonmarine deposits: Earth-Science Reviews, v. 98, no. 3-4, p. 217-268. [Also available at https://doi.org/10.1016/j.earscirev.2009.11.004.]

Williams, P.J., Barton, M.D., Johnson, D.A., Fontboté, L., de Haller, A., Mark, G., Oliver, N.H.S., and Marschik, R., 2005, Iron oxide copper-gold deposits-Geology, spacetime distribution, and possible modes of origin: Littleton, Colo., Society of Economic Geologists, Inc., Economic Geology 100th Anniversary Volume, p. 371-405.

Williams-Stroud, S., 1991, Descriptive model of iodinebearing nitrate (Model 35bl), in Orris, G.J., and Bliss, J.D., eds., Some industrial mineral deposit models-Descriptive deposit models: U.S. Geological Survey Open-File Report 91-11A, p. 51-52.

Woodruff, L.G., Nicholson, S.W., and Fey, D.L., 2013, A deposit model for magmatic iron-titanium-oxide deposits related to Proterozoic massif anorthosite plutonic suites: U.S. Geological Survey Scientific Investigations Report 2010-5070-K, 47 p., accessed April 18, 2020, at https://pubs.usgs.gov/sir/2010/5070/k.

Wyborn, L.A.I., Heinrich, C.A., and Jaques, A.L., 1994, Australian Proterozoic mineral systems-Essential ingredients and mappable criteria, in Australasian Institute of Mining and Metallurgy Annual Conference, Darwin, Australia, 1994, Proceedings: Darwin, Australia, Australasian Institute of Mining and Metallurgy, p.109-115.

Zachmann, D.W., and Johannes, W., 1989, Cryptocrystalline magnesite, in Moeller, P., ed., Magnesite - Geology, mineralogy, geochemistry, formation of Mg-carbonates: BerlinStuttgart, Borntraeger, p. 15-28.

Zartman, R.E., and Smith, J.V., 2009, Mineralogy and U-Th$\mathrm{Pb}$ age of a uranium-bearing jasperoid vein, Sunshine Mine, Coeur d'Alene district, Idaho, USA: Chemical Geology, v. 261, p. 185-195.

Zientek, M.L., Loferski, P.J., Parks, H.L., Schulte, R.F., and Seal, R.R., II, 2017, Platinum-group elements, chap. N of Schulz, K.J., DeYoung, J.H., Jr., Seal, R.R., II, and Bradley, D.C., eds., Critical mineral resources of the United StatesEconomic and environmental geology and prospects for future supply: U.S. Geological Survey Professional Paper 1802, p. N1-N91, accessed April 18, 2020, at https://doi.org/10.3133/pp1802N. 
Publishing support provided by the Science Publishing Network, Denver Publishing Service Center

For more information concerning the research in this report, contact the Center Director, USGS Geology, Geophysics, and Geochemistry Science Center

Box 25046, Mail Stop 973

Denver, CO 80225

(303) 236-1800

Or visit Geology, Geophysics, and Geochemistry Science Center website at https://www.usgs.gov/centers/gggsc 


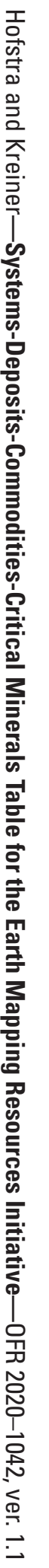

